\title{
Singularities, Black Holes, and Cosmic Censorship: A Tribute to Roger Penrose
}

\author{
Klaas Landsman ${ }^{1}$
}

Received: 8 January 2021 / Accepted: 25 January 2021 / Published online: 31 March 2021

(c) The Author(s) 2021

\begin{abstract}
In the light of his recent (and fully deserved) Nobel Prize, this pedagogical paper draws attention to a fundamental tension that drove Penrose's work on general relativity. His 1965 singularity theorem (for which he got the prize) does not in fact imply the existence of black holes (even if its assumptions are met). Similarly, his versatile definition of a singular space-time does not match the generally accepted definition of a black hole (derived from his concept of null infinity). To overcome this, Penrose launched his cosmic censorship conjecture(s), whose evolution we discuss. In particular, we review both his own (mature) formulation and its later, inequivalent reformulation in the PDE literature. As a compromise, one might say that in "generic" or "physically reasonable" space-times, weak cosmic censorship postulates the appearance and stability of event horizons, whereas strong cosmic censorship asks for the instability and ensuing disappearance of Cauchy horizons. As an encore, an "Appendix" by Erik Curiel reviews the early history of the definition of a black hole.
\end{abstract}

Keywords General relativity $\cdot$ Roger Penrose $\cdot$ Black holes $\cdot$ Ccosmic censorship

Klaas Landsman

landsman@math.ru.nl

1 Department of Mathematics, Radboud University, Nijmegen, The Netherlands 


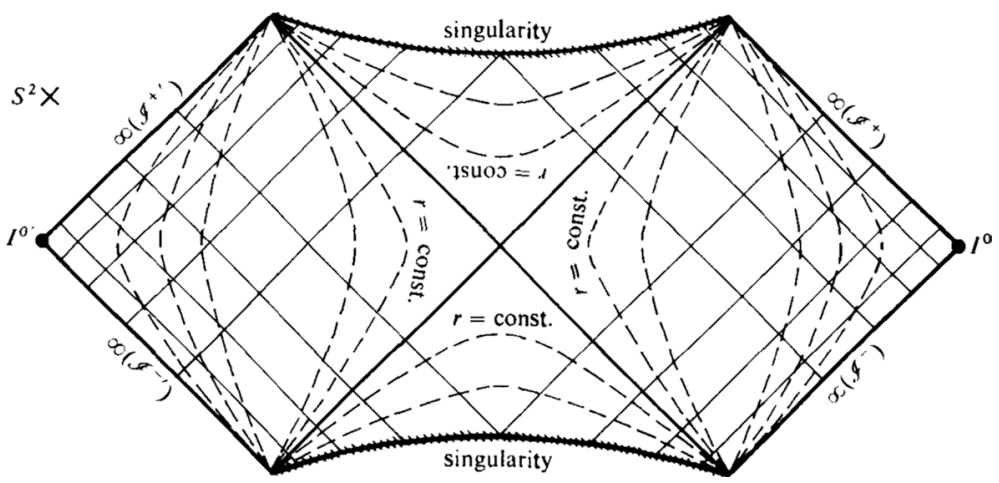

Conformal diagram [146, p. 208, Fig. 37]: 'The Kruskal picture with conformal infinity represented.' Penrose usually drew his own figures in a professional, yet playful and characteristic way.

\section{Historical Introduction}

Roger Penrose got half of the 2020 Physics Nobel Prize 'for the discovery that black hole formation is a robust prediction of the general theory of relativity'. This prize was well deserved, since, jointly with Hawking and others, Penrose has shaped our (mathematical) thinking about general relativity (GR) and black holes since the 1960s-1970s. He would also deserve the Abel Prize for this, shared with Yvonne Choquet-Bruhat: their combination would highlight the fact that two originally distinct traditions in the history of mathematical GR have now converged. In the wake of the work of Einstein [72], these traditions may be said to have originated with Hilbert [100] and Weyl [182, 183], respectively, as follows.

It would be fair to say that Hilbert mainly looked at GR from the point of view of PDES, ${ }^{1}$ whereas Weyl-once Hilbert's PhD student in functional analysis-had a more geometric view, combined with an emphasis on causal structure. These different perspectives initially developed separately, in that the causal theory did not rely on the PDE theory whilst for a long time the PDE results were local in nature. Penrose contributed decisively to the causal approach to GR, with its characteristic emphasis on the conformal structure, ${ }^{2}$ i.e. the equivalence class of the metric tensor $g$ under a rescaling $g_{\mu \nu}(x) \mapsto e^{\lambda(x)} g_{\mu \nu}(x)$, with $\lambda$ an arbitrary smooth function of space and time. Though Weyl [183, p. 397], mentions the analogy with Riemann

\footnotetext{
1 See Stachel [173] for an analysis of Hilbert's contribution, as well as for the history of the Cauchy problem of GR up to Choquet-Bruhat, whose contributions were reviewed by Ringström [157] as well as by herself [27, 28].

${ }^{2}$ Inspired by special rather than general relativity, Robb [158, 159], Reichenbach [154], Zeeman [187], and others axiomatized causal structure as a specific partial order, as Penrose knew well.
} 
surfaces, ${ }^{3}$ his real argument for conformal invariance was that what he calls Reine Infinitesimalgeometrie must go beyond Riemannian geometry, which (so he thinks) suffers from the inconsistency that parallel transport of vectors (through the metric or Levi-Civita connection, a concept Weyl himself had co-invented) preserves their length. This makes length of vectors an absolute quantity, which a 'pure infinitesimal geometry' or a theory of general relativity should not tolerate. To remedy this, Weyl introduced the idea of gauge invariance, in that the laws of nature should be invariant under the above rescaling. To this end, he introduced what we now call a gauge field $\varphi=\varphi_{\mu} d x^{\mu}$ and a compensating transformation $\varphi_{\mu}(x) \mapsto \varphi_{\mu}(x)-\partial_{\mu} \lambda(x)$, and identified $\varphi$ with the electromagnetic potential (i.e. $A$ ). Dancing to the music of time, he then proposed that the pair $(g, \varphi)$ describes all of physics. The idea of gauge symmetry has lasted and forms one of the keys to modern high-energy physics and quantum field theory: though misplaced in the classical gravitational context in which he proposed it, ${ }^{4}$ through the Standard Model it has ironically become a cornerstone of non-gravitational quantum physics!

The conformal structure of a Lorentzian manifold determines the light cones, and as such Weyl was not the only author to discuss causal structure. For example, Einstein [73] himself wondered if gravitational waves propagate with the speed of light, and showed this in a linear approximation; Weyl mentions this also. ${ }^{5}$ The themes of gravitational radiation, conformal invariance, and causal order were combined and came to a head in the work of Penrose, ${ }^{6}$ who also received additional inspiration from the Dutch artist M.C. Escher and the spinor theory of Dirac. In Penrose $[142-146,148]$ he introduced most of the global causal techniques and topological ideas that are now central to any serious mathematical analysis of both GR and Lorentzian geometry $[135,139]$. An important exception is global hyperbolicity, which has its roots in the work of Leray [123] and was adapted to GR by Choquet-Bruhat [25] and Geroch [87]. Global hyperbolicity is the main concept through which the causal theory meets the PDE theory, but Penrose hardly worked on the PDE side. ${ }^{7}$

The second piece of history one needs in order to understand Penrose's contributions that are relevant to his Nobel Prize, is astrophysical. Briefly: ${ }^{8}$ relatively light stars retire as white dwarfs, in which nuclear burning has ended and inward gravitational pressure is stopped by a degenerate electron gas. In 1931 Chandrasekhar

\footnotetext{
${ }_{3}^{3}$ Riemann surfaces may equivalently be defined as either one-dimensional complex manifolds or as twodimensional Riemannian manifolds up to conformal equivalence. Modestly, Weyl does not cite his own decisive contribution to their theory [181]. This equivalence undoubtedly also influenced Penrose's work on GR and its derivatives like twistor theory.

${ }^{4}$ See Einstein's negative reaction to Weyl [183] in Einstein [74, Doc. 8]. See also Goenner [93, §4.1.3].

${ }^{5}$ See e.g. p. 251 of the English translation of the fourth edition of Raum - Zeit - Materie (Weyl, 1922).

${ }^{6}$ This history largely remains to be written (Dennis Lemkuhl is working on this). For now, see e.g. Thorne [175], Frauendiener [81], Friedrich [83], Wright [184, 185], and Ellis [76]. Furthermore, both the written AIP interview by Lightman [125] and the videotaped interview by Turing's biographer Hodges [103] are great and intimate portraits of Penrose.

${ }^{7}$ Although he was well aware of it: the singularity theorem in Penrose [144] assumes the existence of a Cauchy surface.

${ }^{8}$ See Israel [109], Luminet [130], Thorne [175], Melia [133], Sanders [165], Curiel [49], and Falcke [78] for history, and Misner et al. [136], Joshi [110, 111], Poisson [153], and Weinberg [180] for theory.
} 
discovered that this works only for masses $m \leq 1.46 M_{\odot}$, where $M_{\odot}$ is the solar mass. Heavier stars collapse into neutron stars (typically after a supernova explosion), but also these have an upper bound on their mass, as first suggested by Oppenheimer and Volkoff (1939); the current value is about $2.3 M_{\odot}$. Stars that are more massive cannot stop their gravitational collapse and unless they get rid of most of their mass/ energy they collapse completely. ${ }^{9}$ But what does this mean mathematically?

Most of the early intuition came from the Schwarzschild solution, seen as a model of the final state of such a collapse. This solution is spherically symmetric, and by Birkhoff's theorem any such vacuum solution must be Schwarzschild (or Minkowski). It has two very notable features, namely curvature singularity as $r \rightarrow 0$ and an event horizon at $r=2 \mathrm{~m}$. Here it should be mentioned that initially both caused great confusion, even among the greatest scientists involved such as Einstein and Hilbert, though Lemaître was ahead of his time. ${ }^{10}$ Apart from their exact locations, these two features, then, may be taken to be the defining characteristics of a black hole, but especially the event horizon, which is held to be responsible for the "blackness" of the "hole". However, even short of a correct technical understanding of these features, from the 1920s until the 1950s most leading researchers in GR (including Einstein, Eddington, as well as Landau's school in the Soviet Union, which covered all of theoretical physics) felt that at least the singularity was an artifact of the perfect spherical symmetry of the solution (and likewise for the big bang as described by the spherically symmetric Friedman/FLRW solution). This negative view also applied to the first generally relativistic collapse model (Oppenheimer and Snyder, 1939), now seen as groundbreaking, which is spherically symmetric and therefore terminates in the Schwarzschild solution.

The achievement usually attributed to Penrose [144], culminating in his Nobel Prize, is that he settled (in the positive) the question whether a more general (i.e. non-spherically symmetric) collapse of sufficiently heavy stars (etc.) also leads to a black hole. But if anyone understood this was not the case for the construal of a black hole as an astrophysical object with event horizon, it was Penrose himself! He must have been the first to recognize that his singularity theorem from 1965 did not prove the existence of black holes; under suitable hypotheses (involving both the concentration of matter and the causal structure of space-time) it proved merely the existence (but not even the precise nature or location) of incomplete null geodesics. As such, this implies neither the existence of a curvature singularity (not even if the solution is close to Schwarzschild), nor that of an event horizon. Leaving the former aside for the moment, the latter became the topic of what is now called the weak cosmic censorship conjecture: ${ }^{11}$

\footnotetext{
9 Supermassive black holes like Sagittarius A* and M87* are probably formed by mergers and accretion rather than collapse.

10 See Tipler et al. [176], Godart [92], Eisenstaedt [75], Thorne [175], Earman [66, 68], and Earman and Eisenstaedt [71]. Our understanding of the event horizon as a one-way membrane is usually attributed to Finkelstein [79].

11 It is worth stressing that Penrose included a genericity restriction right from the beginning, pace Dafermos [52, p. 55]. The emphasis on initial data in the second formulation does not recur in the strong version of cosmic censorship below, but it is unavoidable in any form of weak cosmic censorship in order
} 
We are thus presented with what is perhaps the most fundamental question of general-relativistic collapse theory, namely: does there exist a "cosmic censor" who forbids the appearance of naked singularities, clothing each one in an absolute event horizon? In one sense, a "cosmic censor" can be shown not to exist. For it follows from a theorem of Hawking that the "big bang" singularity is, in principle, observable. But it is not known whether singularities observable from outside will ever arise in a generic collapse which starts off from a perfectly reasonable nonsingular initial state. [147, p. 1162]

A system which evolves, according to classical general relativity with reasonable equations of state, from generic non-singular initial data on a suitable Cauchy hypersurface, does not develop any spacetime singularity which is visible from infinity. [149, p. 618]

Following Penrose [149] we give a precise mathematical version in Sect. 2, but in any case it should be clear that in order to prove the existence of black holes from suitable assumptions one needs both Penrose's singularity theorem (which gives at least some kind of singularity) and the weak cosmic censorship hypothesis (which gives the event horizon): the latter is the missing link between theorem and reality.

Expanding the scope of cosmic censorship, Penrose [149, p. 619], subsequently argued that:

It seems to me to be comparatively unimportant whether the observer himself can escape to infinity. Classical general relativity is a scale-invariant theory, so if locally naked singularities occur on a very tiny scale, they should also, in principle, occur on a very large scale in which a 'trapped' observer could have days or even years to ponder upon the implications of the uncertainties introduced by the observations of such a singularity. (...) Indeed, for inhabitants of recollapsing closed universes (as possibly we ourselves are) there is no 'infinity', so the question of being locally 'trapped' is one of degree rather than principle. It would seem, therefore, that if cosmic censorship is a principle of Nature, it should be formulated in such a way as to preclude such locally naked singularities.

This ban is called strong cosmic censorship, which as first shown by Penrose [149] himself, comes down to the requirement of global hyperbolicity (see Sect. 3). However: global hyperbolicity of which space-time?

More generally, in Penrose's singularity theorem as well as in his two versions of cosmic censorship, it is ambiguous to which space-times the theorem and the hypothesis are applied. Traditionally, in GR one typically studied analytically extended solutions to the Einstein equations like-in the context of black holes- the Kruskal extension of the Schwarzschild solution, and similarly (but now not doubled

Footnote 11 (continued)

to exclude the naked big bang singularity from the conjecture: the point of the second (1979) formulation is that the singularity lies to the future of the Cauchy hypersurface in question. 
but "infinitely extended") for Reissner-Nordström and Kerr. The PDE approach to GR, on the other hand, is based on two slogans, appealing to the fundamental theorem of Choquet-Bruhat and Geroch [29]: ${ }^{12}$

- All valid assumptions are about initial data;

- All valid questions are about the maximal globally hyperbolic development (MGHD) thereof.

This clearly affects strong cosmic censorship, in that asking that a physically reasonable space-time be globally hyperbolic is now deemed empty, since the MGHD of any initial data automatically has this property. For similar reasons also Penrose's version of weak cosmic censorship needs to be reformulated. His singularity theorem does make sense for both traditional solutions and MGHD, but the cause of geodesic incompleteness is quite different is these two cases (except for the Kruskal solution).

Written about and by a mathematician, we start in Sect. 2 with definitions. In Sect. 3 we trace the evolution of Penrose's idea of cosmic censorship, which is illustrated by three black hole examples in Sect. 4. In the last section Sect. 5, the whole story culminates in Penrose's amazing and influential final state conjecture. The conclusion is that although arguably Penrose did not quite achieve what the Nobel Prize committee says, he developed most of the techniques, saw the need for singularity theorems (of which he proved the first) as well as cosmic censorship, and, perhaps most importantly, showed the way to others. ${ }^{13}$ Finally, an Appendix written by Erik Curiel traces the definitional history of the concept of a black hole.

\footnotetext{
${ }^{12}$ A Cauchy surface in a space-time $(M, g)$ is a subset $\Sigma \subset M$ such that each endless timelike curve intersects $\Sigma$ exactly once. This makes $\Sigma$ a closed connected $3 d$ submanifold of $M$ which can be chosen space-like and hence Riemannian. A space-time is globally hyperbolic if it has a Cauchy surface. Non-characteristic initial values for the Einstein equations form a triple $(\Sigma, h, K)$, i.e. a $3 d$ Riemannian manifold $(\Sigma, h)$ equipped with an additional symmetric tensor $K_{i j}$, satisfying four constraint equations. A Cauchy development of such initial data is a triple $(M, g, i)$, where $(M, g)$ is a $4 d$ space-time solving the Einstein equations, and $i: \Sigma \rightarrow M$ is an embedding such that $i^{*} g=h$ and $i(\Sigma)$ is a Cauchy surface in $M$ with extrinsic curvature $K$. Hence $(M, g)$ is globally hyperbolic. Choquet-Bruhat and Geroch [29] showed that there exists a Cauchy development of the given $(\Sigma, h, K)$, called the maximal globally hyperbolic development (MGHD), that is maximal as a globally hyperbolic space-time solving the Einstein equations with Cauchy surface $i(\Sigma)$ and given $(h, K)$. This MGHD is unique up to time-orientation-preserving isometries preserving $\Sigma$, i.e. if $(M, g, i)$ and $\left(M^{\prime}, g^{\prime}, i^{\prime}\right)$ qualify then there is an isometry $\psi: M \rightarrow M^{\prime}$ such that $\psi \circ i=i^{\prime}$. See Choquet-Bruhat [26] and Ringström [156] for introductions to the PDE approach, supplemented by Sbierski [166]. See also [41]

${ }^{13}$ In particular, using the PDE approach Christodoulou [31, 33, 34] finally established the formation of black holes both in spherically symmetric collapse models with (scalar field) matter and in vacuum solutions through focusing of gravitational waves, by proving both causal geodesic incompleteness and the existence of an event horizon. See also follow-ups by Klainerman and Rodnianski [116] and Klainerman et al. [114], and reviews by Bieri [12] and Dafermos [52]. For the incorporation of more realistic matter models see e.g. Burtscher and LeFloch [14] and Burtscher [13].
} 


\section{Definitions}

In mathematical physics it is essential to start from definitions that are physically relevant, mathematically precise, and workable. Penrose had a remarkable gift for this. ${ }^{14}$ For our purpose, i.e. what to make of the citation for his Nobel Prize, Penrose contributed at least five great definitions to GR, namely of:

- Null infinity, in turn implying a definition of an event horizon and hence of a black hole;

- Trapped surfaces, formalizing the condition that gravity is strong enough to focus light-rays;

- Singularities in space-time, which he characterized through incomplete causal geodesics;

- Weak cosmic censorship, stating that space-time singularities are covered by event horizons;

- Strong cosmic censorship, forbidding even nearby causal contact with spacetime singularities.

In this section we explain the first three definitions, leaving the last two for a separate section (Sect. 3). ${ }^{15}$

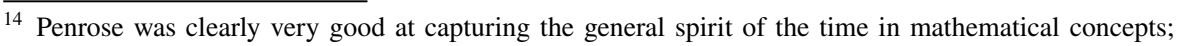
this is why his ideas so quickly became mainstream, despite the unfamiliarity of even theoretical physicists at the time with a field like topology (see [175, Chap. 13], which describes Penrose's role in the GR community). But he did so in his own unique individual way: 'It was important for me always, if I wanted to work on a problem, to think I had a different angle on it from other people. Because I wasn't good at following where everybody else went. I wasn't the kind of person who could pick up the prevalent arguments and knowledge of the time. Other people were good at that. They could suck it all out and put it together and make advances. I was the kind of person who'd have some kind of quirky way of looking at something on my own, which I would hide away and work at. So it meant that I had to have some way of looking at a problem that was my own' [125].

${ }^{15}$ Unexplained notions may be found in the standard GR textbooks such as Wald [179] or Chruściel [38]. A space-time $(M, g)$ is a $4 d$ connected time-orientable Lorentzian manifold with time orientation, i.e. the metric has signature $(-+++)$ and one has a way of distinguishing past from future by selecting, at each point in a continuous and consistent way, a forward and a backward light-cone. This leads to one of Penrose's most effective notations, namely the relation $J \subset M \times M$, where $(x, y) \in J$, also written as $y \in J^{+}(x)$ or $x \in J^{-}(y)$ or $x \leq y$, iff there exists a future-directed (fd) causal curve from $x$ to $y$. For $A \subset M$ we write $J^{ \pm}(A)=\cup_{x \in A} J^{ \pm}(x)$. Similarly, $I \subset M \times M$ is defined by replacing 'causal' by 'timelike'; one writes $x \ll y$ iff $(x, y) \in I$.
} 


\subsection{Null Infinity}

Null infinity and the ensuing concept of a black hole are predicated on the following concept: ${ }^{16}$

\section{Definition 2.1}

1. A conformal completion of a space-time $(M, g)$ is a space-time $(\tilde{M}, \tilde{g})$, where $\tilde{M}$ is a manifold with boundary, with an embedding $l: M \hookrightarrow \tilde{M}$ such that $\iota(M)=\operatorname{int} \tilde{M}):=\tilde{M} \backslash \partial \tilde{M}$, and $\iota$ is conformal in that $\iota^{*} \tilde{g}=\left(\imath^{*} \Omega^{2}\right) g$ for some smooth positive function $\Omega: \tilde{M} \rightarrow \mathbb{R}^{+}$that satisfies:

$$
\Omega>0 \text { on } \imath(M) ; \quad \Omega=0 \text { on } \partial \tilde{M} ; \quad \tilde{\nabla} \Omega \neq 0 \text { on } \partial \tilde{M} .
$$

2. $(M, g)$ is asymptotically flat at null infinity if it has a conformal completion $(\tilde{M}, \tilde{g})$ for which:

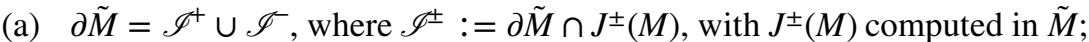

(b) The Ricci tensor of the original metric $g$ is such that $R_{\mu \nu}=O\left(\Omega^{3}\right)$ pointwise near $\partial \tilde{M}$.

In clause 2 and in what follows we tacitly identify $M$ with $t(M)$. Here are some comments on this clause.

2(a) The boundary $\mathscr{I}$ (pronounced, as Penrose suggests, "scri") is called null infinity. Its components $\mathscr{I}^{+}$and $\mathscr{I}^{-}$are called future null infinity and past null infinity, respectively. The idea is that $\mathscr{I}^{+}\left(\mathscr{I}^{-}\right)$consists of limit points of future (past) directed null curves along which $r \rightarrow \infty$.

2(a) Asking $O\left(\Omega^{3}\right)$ is on the safe side (one might ask $O\left(\Omega^{2+\varepsilon}\right)$ for $1 / 2<\varepsilon \leq 1$ ), and implies that $\Omega^{-2} R_{\mu \nu}$ extends by continuity from $l(M)$ to zero on $\partial \tilde{M}$, as in $R_{\mu \nu}(r) \sim 1 / r^{3}$ as $r \rightarrow \infty$. The simplest way to satisfy this is to assume that $(M, g)$ solves the vacuum Einstein equations $R_{\mu \nu}=0$; in the presence of matter one equivalently asks that $T_{\mu \nu}$ be $O\left(\Omega^{3}\right)$.

\footnotetext{
${ }^{16}$ See originally Penrose [143], who-in the context of gravitational waves-adds the condition that every null geodesic has two end-points on $\partial \tilde{M}$, defining $(M, g)$ to be asymptotically simple. In that case each connected component of $\partial \tilde{M}$ is diffeomorphic to $\mathbb{R} \times S^{2}$, as is often the case even more generally (and as such is sometimes included in Definition 2.1). See also Hawking and Ellis [98, §6.9], Geroch [88], Wald [179, §11.1], Penrose and Rindler [152, Chap. 9], Stewart [174, Chap. 3], Frauendiener [81], Valiente Kroon [177], and Chruściel [39, §3.1]. The question how Definition 2.1 relates to asymptotic flatness as defined through conditions on the metric, either in space-time $(4 d)$ or in the initial value problem $(3 d)$, is very subtle; smoothness of $(\tilde{M}, \tilde{g})$ implies detailed fall-off (or 'peeling') properties of the Weyl tensor at infinity. See e.g. Geroch [88], Stewart [174], Klainerman and Nicolò [115], Friedrich [82, 84], Adamo et al. [1], Dafermos [52], Chruściel and Paetz [45], and Paetz [141]. However, for the usual stationary black hole solutions and more generally for stationary space-times satisfying standard energy conditions the boundary is smooth [40].
} 
A crucial fact, noted (mutatis mutandis) without proof in Penrose [143, 146], is that: ${ }^{17}$

Proposition 2.2 On the boundary $\partial \tilde{M}$ the scaling function $\Omega$ satisfies the eikonal equation

$$
\tilde{g}(\tilde{\nabla} \Omega, \tilde{\nabla} \Omega)=0,
$$

so that $\partial \tilde{M}$ (more precisely: each connected component thereof) is a null hypersurface in $\tilde{M} .^{18}$

Proof A simple computation, based on a conformal rescaling of the Ricci tensor, ${ }^{19}$ shows that

$$
\tilde{g}(\tilde{\nabla} \Omega, \tilde{\nabla} \Omega)=\frac{1}{12}\left(\Omega^{2} \tilde{R}-R\right)+\frac{1}{2} \Omega \Delta_{\tilde{g}} \Omega .
$$

Since $\tilde{g}$ is regular on $\partial \tilde{M}$ (where $\Omega=0$ ) and $R_{\mu \nu}=O\left(\Omega^{3}\right.$ ) gives $R=O(\Omega)$, Eq. (2.2) follows.

Following Hawking and Penrose-we leave the tangled history to the Appendix-we may then define

$$
\mathscr{B}:=M \backslash J^{-}\left(\mathscr{I}^{+}\right) ; \quad \mathscr{W}:=M \backslash J^{+}\left(\mathscr{I}^{-}\right),
$$

called the black hole region and the white hole region in $M$, respectively; each connected component of $\mathscr{B}$, if not empty, is then simply a black hole. ${ }^{20}$ It can be shown that $J^{\mp}\left(\mathscr{I}^{ \pm}\right)$is open. ${ }^{21}$ The boundaries

$$
\mathscr{H}_{E}^{+}:=\partial \mathscr{B} ; \quad \mathscr{H}_{E}:=\partial \mathscr{W}
$$

\footnotetext{
${ }_{17}$ If $R_{\mu \nu}=\lambda g_{\mu \nu}$, then $\tilde{g}(\tilde{\nabla} \Omega, \tilde{\nabla} \Omega)=-\frac{1}{3} \lambda$ on $\partial \tilde{M}$, so that $\tilde{\nabla} \Omega$ is timelike and hence $\partial \tilde{M}$ is spacelike if $\lambda>0$, and vice versa if $\lambda<0$ [143, Lecture II], [146, p. 181]. See Ashtekar et al. [6] and Ashtekar and Magnon [8], respectively, for these cases. But as the king of null geometry in GR, Penrose must have taken special pleasure in $\lambda=0$ !

${ }^{18}$ Short of the very subtle regularity issues discussed in footnote 16 , the boundary $\mathscr{I}$ is smooth, and points like $i^{ \pm}$and $i^{0}$, typically included in Penrose diagrams, are not part of it. However, if one is interested in spatial infinity $[4,5,88]$ one could extend the definition of a conformal completion so as to include these points.

${ }^{19}$ It is easily verified by direct computation, and found in many books [177, §5.2.2], [39, Appendix H.6] that if $g^{\prime}=\varphi^{2} g$, then $R_{\mu \nu}^{\prime}=R_{\mu \nu}-\varphi^{-1}\left(2 \nabla_{\mu} \nabla_{\nu} \varphi+g_{\mu \nu} \Delta_{g} \varphi\right)+\varphi^{-2}\left(4 \nabla_{\mu} \varphi \nabla_{\nu} \varphi-g_{\mu \nu} g(\nabla \varphi, \nabla \varphi)\right)$. Now replace $g^{\prime} \rightsquigarrow g$ and $g \rightsquigarrow \tilde{g}$, so that $\varphi=1 / \Omega$. This gives $R_{\mu \nu}=\tilde{R}_{\mu \nu}+\Omega^{-1}\left(2 \tilde{\nabla}_{\mu} \tilde{\nabla}_{\nu} \Omega+\tilde{g}_{\mu \nu} \Delta_{\tilde{g}} \Omega\right)-3 \Omega^{-2} \tilde{g}(\tilde{\nabla} \Omega, \tilde{\nabla} \Omega) \tilde{g}_{\mu \nu}$, where $\tilde{\nabla}=\tilde{g}^{\rho \sigma} \tilde{\nabla}_{\rho} \tilde{\nabla}_{\sigma}$. This is eq. (11.1.16) in Wald [179], which immediately yields (2.3).

${ }^{20}$ See the Appendix by Erik Curiel for historical information on this definition. See also Thorne [175, Chap. 7].

${ }^{21}$ Since $I^{\mp}\left(\mathscr{I}^{ \pm}\right) \cap M=J^{\mp}\left(\mathscr{I}^{ \pm}\right) \cap M$, one could have used $I$ instead of $J$ in (2.4); see Wald [179, p. 308].
} 
decompose into the future and past event horizons of each of the black and white holes in $M$ respectively. Since the hole regions $\mathscr{B}$ and $\mathscr{W}$ are closed, the event horizons form part of the black/white holes.

The analysis of such space-times is greatly facilitated by Penrose's conformal diagrams, ${ }^{22}$ now called Penrose diagrams. ${ }^{23}$ These became an important tool for visualizing black holes $[20,98]$. The title page shows one of the first such diagrams, drawn by Penrose himself.

However, the above definition of a black hole, though mathematically sweet, is not uncontroversial:

This definition depends on the whole future behaviour of the solution; given the partial Cauchy surface $\mathscr{S}(\tau),{ }^{24}$ one cannot find where the event horizon is without solving the Cauchy problem for the whole future development of the surface.' (Hawing and Ellis, 1973, p. 319)

[The future event horizon] is the boundary of an interior spacetime region from which causal signals can never be sent to the asymptotic observers, no matter how long they are prepared to wait. The region is therefore "black" in an absolute sense.' [7, p. 2]

The idea that nothing can escape the interior of a black hole once it enters makes implicit reference to all future time-the thing can never escape no matter how long it tries. Thus, in order to know the location of the event horizon in spacetime, one must know the entire structure of the space-time, from start to finish, so to speak, and all the way out to infinity. As a consequence, no local measurements one can make can ever determine the location of an event horizon. That feature is already objectionable to many physicists on philosophical grounds: one cannot operationalize an event horizon in any standard sense of the term. Another disturbing property of the event horizon, arising from its

\footnotetext{
${ }^{22}$ These confirm what Penrose often says, namely that he prefers to think in terms of pictures. Since Penrose started in algebraic geometry as a $\mathrm{PhD}$ student of Hodge in Cambridge, he was undoubtedly influenced by the theory of Riemann surfaces in finding this concept (like Weyl, as mentioned in the historical introduction). For example, mutatis mutandis the closed Poincare disk is a Penrose diagram of the Poincaré upper half plane. Penrose must also have been influenced by the famous Circle Limit woodcuts by Escher (nos. I-IV, dating from 1958 to 1960). See also Wright [184, 185].

23 Or, sometimes, Penrose-Carter diagrams. Carter himself speaks of $P C$-diagrams, perhaps tongue-incheek saying that $P C$ stands for Projective Conformal. See Chruściel [39, Chap. 6] for an axiomatic theory of such diagrams. On a pragmatic case-by-case basis, construct and draw $\tilde{M}$, suppress two-spheres, and use coordinates in which null geodesics are at $\pm 45^{\circ}$, as in Minkowski space-time (indeed the $\pm 45^{\circ}$ idea goes back to Minkowski himself, who also drew his own diagrams).

24 A partial Cauchy surface $\Sigma$ is an acausal edgeless subset of $M$ [98, p. 204], [135, , p. 95]. This makes $\Sigma$ a closed hypersurface in $M$ which, because it is edgeless, is inextendible as an acausal set (though not necessarily maximal in $M$ as such). A sufficient condition for the existence of a partial Cauchy surface is the existence of a time function; see Minguzzi [135, Theorems 3.39 and 4.100]. In the PDE approach it arises when strong cosmic censorship fails, see Sect. 3.2, and a Cauchy surface for the MGHD turns into a partial one for the extension. In that case $\Sigma$ acquires a non-empty Cauchy horizon $H_{C}(\Sigma)=\partial D(\Sigma)$, where $D(\Sigma)$ is the domain of dependence of $\Sigma$, splitting into past and future ones $H_{C}(\Sigma)=H_{C}^{-}(\Sigma) \cup H_{C}^{+}(\Sigma)$.
} 
global nature, is that it is prescient. Where I locate the horizon today depends on what I throw in it tomorrow-which future-directed possible paths of particles and light rays can escape to infinity starting today depends on where the horizon will be tomorrow, and so that information must already be accounted for today. Physicists find this feature even more troubling. [50, p. 29]

It is amusing how differently even top GR experts (and textbook authors!) respond to this charge:

[The above definition of an event horizon] is probably very useless, because it assumes we can compute the future of real black holes, and we cannot. (Rovelli, quoted in [50, p. 30])

I have no idea why there should be any controversy of any kind about the definition of a black hole. There is a precise, clear definition in the context of asymptotically flat spacetimes (...) I don't see this as any different than what occurs everywhere else in physics, where one can give precise definitions for idealized cases but these are not achievable/measurable in the real world. (Wald, ibid., p. 32)

What seems at stake here is what may be called Earman's Principle:

While idealizations are useful and, perhaps, even essential to progress in physics, a sound principle of interpretation would seem to be that no effect can be counted as a genuine physical effect if it disappears when the idealizations are removed. [69, p. 191]

Note that two kinds of idealizations are involved in the case of event horizons of black holes:

1. The ability to know an entire space-time $(M, g)$, either from initial data or by direct construction;

2. The construction of null infinity in terms of which black holes and event horizons are defined.

Rovelli's comment seems to apply to the first point but Wald's to the second, in which case they would not contradict each other. The need to idealize the idea (!) that an event horizon prevents sending signals from the singularity to observers "far away" by taking the latter to mean "at (null) infinity" arises because in general any finite distance could potentially lie within the event horizon. For specific space-times like Kruskal or Kerr, the horizons $\mathscr{H}_{E}^{ \pm}$as defined in (2.5) can be explicitly located in $M$ without reference to (null) infinity. ${ }^{25}$ Even if the space-time is not

\footnotetext{
25 This is different from the idealization in phase transitions and spontaneous symmetry breaking, where even in exactly solvable models one needs the idealization of the thermodynamic limit to have such effects, at least according to their official definition. See Butterfield [15] and [122, Chap. 10] for the way to deal with Earman's principle in these cases.
} 
known explicitly, the event horizon (if it has one) by definition lies at some finite distance from the singularity (if it has one). Hence in locating the horizon, the phrase "at infinity" could be replaced by "sufficiently far away" (from the singularity), which agrees with Earman's principle_and therefore Wald's stance seems valid provided it concerns the second point.

On the other hand, the second point is predicated on the first, which remains unresolved. Thus we are entering an almost axiomatic approach to GR here, liable to the famous charge that it has 'the advantages of theft over honest toil' [163, p. 71]. However, nothing is wrong with an axiomatic approach as long as one can find realistic models for the axioms (or definitions) that show that they are reasonable. This is the case in Penrose's approach. The fact that we cannot 'compute the future of black holes' does not disqualify the event horizon as an object of nature we can prove theorems about (whose desirability may be different for theoretical and mathematical physicists). What $i s$ worrying is the precise relationship between the black hole "shadow" in the EHT image of M87* and the event horizon as defined by (2.5), which we cannot possibly know now. ${ }^{26}$ This raises epistemological questions about the role of theory in observation, which will not even be addressed here, let alone answered. See also Franklin [80].

\subsection{Trapped Surfaces}

In their excellent review of Penrose's 1965 singularity theorem, Senovilla and Garfinkle [171] explain that all singularity theorems in GR share the following three assumptions (we quote verbatim):

(i) a condition on the curvature;

(ii) a causality condition;

(iii) an appropriate initial and/or boundary condition.

In Penrose [144] condition (i) states that $R_{\mu \nu} \dot{\gamma}^{\mu} \dot{\gamma}^{v} \geq 0$ along all null geodesics $\gamma$. Condition (ii) states that the space-time be globally hyperbolic with non-compact Cauchy surface; the topological assumption reflects the idea that the theorem is supposed to apply to black holes and hence to asymptotically flat space-times. His condition (iii) is the existence of a closed trapped surface, which is one of the most important concepts in all of black hole (mathematical) physics. ${ }^{27}$ Here is Penrose's own definition:

\footnotetext{
${ }^{26}$ More precisely, where it was 53.5 million years ago. An additional complication is that (ignoring the rotation of $\mathrm{M} 87^{*}$ for simplicity) the edge of the disk is not the event horizon at $r=2 m$ but the photon sphere at $r=3 m$, further dislocated by optical effects so that we actually see it at $r=\sqrt{27} \mathrm{~m}$, cf. Chruściel [38, §3.9.6], and Event Horizon Telescope Collaboration [77].

27 See initially Hawking [98, Chap. 9]. The study of trapped surface formation from the PDE point of view began with Schoen and Yau [169], who gave initial values that already contain trapped surface; see also Alaee et al. [2]. Christodoulou [31, 32, 34] first proved the evolution of asymptotically flat initial data into trapped surfaces. Later literature may be traced back from Li and Yu [124] and Athanasiou and Lesourd [9]. See also references in Footnote 13.
} 
A trapped surface [is] defined generally as a closed, spacelike two-surface $T^{2}$ with the property that the two systems of null geodesics which meet $T^{2}$ orthogonally converge locally in future directions at $T^{2}$. [144, p. 58]

In the presence of a radial coordinate $r$ as in the Schwarzschild, Reissner-Nordström, and Kerr solutions, this condition is equivalent to the (metric) gradient $\nabla r$ being timelike, which in the Schwarzschild solution happens for $r<2 m$, and which in the other two (subcritical) cases is the case at least for a while after crossing the event horizon. In general, the convergence condition can be stated in terms of the null hypersurface $C$ generated by the future directed null congruence emanating from some (instantaneous) spacelike two-sphere $S^{2}$, so that $\partial C=S^{2}$. In terms of a tetrad $\left(e_{1}, e_{2}, L, \underline{L}\right)$ with $\left(e_{1}, e_{2}\right)$ spacelike and tangent to $C, L$ null and tangent as well as orthogonal to $C$, and $\underline{L}$ null and pointing off $C$, normalized such that $g\left(e_{i}, e_{j}\right)=\delta_{i j}$, $g\left(e_{i}, L\right)=g\left(e_{i}, \underline{L}\right)=0$ for $i, j=1,2$, and finally $g(L, \underline{L})=-1$ and of course $g(L, L)=g(\underline{L}, \underline{L})=0$, all defined on $C$, null extrinsic curvatures are $2 \times 2$ matrices $k_{i j}=g\left(\nabla_{j} L(\bar{t}), \bar{e}_{i}(t)\right)$ and $\underline{k}_{i j}=g\left(\nabla_{j} \underline{L}, e_{i}\right)$, with traces $\theta=\operatorname{tr}(k)$ and $\underline{\theta}=\operatorname{tr}(\underline{k})$. Then $S^{2}$ is trapped iff $\theta<0$ and $\underline{\theta}<0$ throughout $S^{2}$. This condition is local and there are none of the problems afflicting null infinity (cf. Sect. 2.1).

\subsection{Singularities in Space-Time}

Ironically, although this is also seen as one of Penrose's most important contributions to GR (and was immediately recognized as such by his contemporaries like Hawking), his definition of a singular space-time (i.e. as being causally geodesically incomplete) has to be inferred from his proof by contradiction of his singularity theorem in Penrose [144, 146], to the effect that properties (i), (ii), and (iii) of the previous subsection exclude the possibility that $(M, g)$ is also future null geodesically complete. ${ }^{28}$

Hawking [95, §6.1], much more explicitly discusses the need for a good definition of a singular space-time, upon which he arrives at the contrapositive: 'We shall say that $M$ is singularity-free if and only if it is timelike and null geodesically complete. ${ }^{29}$ However, unlike Penrose [144, 146], Hawking, and later Hawking and Ellis $[98, \S 8.1]$, emphatically apply this definition to the case where $(M, g)$ is metrically inextendible, in that it cannot be isometrically embedded as an open submanifold of a larger space-time $\left(M^{\prime}, g^{\prime}\right)$, subject to certain regularity conditions on both the manifold and the metric. Adapting a definition given by Clarke [46, p. 10] (who however uses arbitrary curves) we may formalize this by:

\footnotetext{
${ }^{28}$ A geodesic $\gamma$, which we take by definition to be affinely parametrized, is called complete if it can be extended to arbitrary values of its parameter, i.e. is defined as a map $\gamma: \mathbb{R} \rightarrow M$. It is future complete if it is defined as a map $\gamma:[a, \infty) \rightarrow M$ for some $a \in \mathbb{R}$, etc. In the Riemannian case, by the Hopf-Rinow theorem geodesic completeness is equivalent to completeness in the topological metric $d$ derived from the Riemannian metric $g$ as the infimum over the path length (computed from $g$ ) of all curves connecting two given points. Since a Lorentzian metric no longer defines a topological metric, this result is lost.

29 As in Hawking and Ellis [98, §8.1], Penrose is not mentioned here but there is generic acknowledgement in the Preface.
} 
Definition 2.3 A space-time is singular if it contains an incomplete causal geodesic $\gamma:[0, a) \rightarrow M$ such that there is no extension $\theta: M \rightarrow M^{\prime}$ for which $\theta \circ \gamma$ is extendible.

This refinement of Penrose's definition was originally proposed in order to avoid trivial cases: removing any point from Minkowski space-time makes it geodesically incomplete, but also think of Schwarzschild for $r>2 m$ only. But with hindsight, we can say it makes a big difference to impose inextendibility also in nontrivial cases where strong cosmic censorship fails, as will be explained in due course. We therefore follow Penrose in defining a space-time to be singular iff it is causally geodesically incomplete, leaving it open whether it can be extended-indeed his 1965 singularity theorem (or any later version thereof) gives no information about metric inextendibility at all. As we shall see, if the three conditions in Penrose's singularity theorem hold, the cases where the space-time in question can or cannot be extended are quite different in so far as the nature of the incompleteness is concerned, and both cases are equally interesting. Even apart from this, Penrose's definition is once again controversial; it ended a long period of confusion, but it did so at a price, as was recognized right from the start. As Geroch $[86],{ }^{30}$ p. 526, states:

(a) there is no widely accepted definition of a singularity in general relativity;

(b) each of the proposed definitions is subject to some inadequacy.

For example, the link between singularities and diverging curvature is lost, although this was the original intuition from both the Schwarzschild and the Friedman "singularities". Furthermore, even within the confines of defining singularities through incomplete curves, singling out (causal) geodesics excludes some interesting space-times intuitively felt to be singular-but this can only be detected through the incompleteness of more general curves (Geroch [86, Appendix]). In fact, Penrose [149] did incorporate these at a later stage, as will be discussed in Sect. 3.1. But ultimately we side with Hawking and Ellis: ${ }^{31}$

Timelike geodesic completeness has an immediate physical significance in that it presents the possibility that there could be freely moving observers or particles whose histories did not exist after (or before) a finite interval of proper time. This would appear to be an even more objectionable feature than infinite curvature and so it seems appropriate to regard such a space as singular. (...) The advantage of taking timelike and/or null incompleteness as being indicative of the presence of a singularity is [also] that on this basis one can establish a number of theorems about their occurrence. [98, p. 258]

\footnotetext{
${ }^{30}$ Further to this classical paper on singularities, see also Earman [66, 67], Senovilla [170], and Curiel $[48,49]$.

${ }^{31}$ Reminiscent of the great slogan 'A good definition should be the hypothesis of a theorem' (attributed to J. Glimm).
} 


\section{Cosmic Censorship}

In this section we review Penrose's original versions of cosmic censorship, followed by PDE reformulations now in use. Penrose [149] gave a precise statement of strong cosmic censorship that seems almost forgotten, but translated this into an equivalent characterization in terms of global hyperbolicity that became very influential. Since only seasoned relativists will be able to relate global hyperbolicity to the original ideas behind cosmic censorship (as reviewed in the historical introduction), we first give a unified formulation of both weak and strong cosmic censorship along the lines of Penrose [149], (see also Penrose [150]).

\subsection{Cosmic Censorship à la Penrose}

Remarkably, where Penrose [144] defined singularities in terms of incomplete causal geodesics, Penrose [149] switches to endless timelike curves. It turns out that the change from 'causal' to 'timelike' does not matter, ${ }^{32}$ but the change from geodesics to curves is quite substantial. ${ }^{33}$ Forbidding signaling by singularities thus defined turns out to be equivalent to global hyperbolicity (of all of space-time in case of strong cosmic censorship and of $J^{-1}\left(\mathscr{I}^{+}\right)$in the weak version), which is very neat and may justify this change. However, had the original definition in terms of causal geodesics been used, then presumably some weaker causality condition than global hyperbolicity would have been found. ${ }^{34}$

In any case, the basic problem is to express mathematically what it means for a signal to emanate from a singularity, since the latter is not part of space-time. Happily, it is precisely his own definition of singularities in terms of incomplete causal geodesics-now general causal curves-that enabled Penrose to overcome this problem, drawing on earlier work [90], as follows.

\footnotetext{
32 In the light of the analysis below, this follows from Theorem (2.3) in Geroch et al. [90].

33 Penrose's timelike curves are smooth by convention [148, pp. 2-3]. Following Minguzzi [135], we prefer to work with continuous causal curves, which behave better under limits (e.g. smooth timelike curves typically converge uniformly, if they do, to continuous causal curves, whereas limits of the latter, if they exist, lie in the same class). We say that a continuous curve $c: I \rightarrow M$ is causal if every point $x=c(t)$ on the curve $(t \in I)$ has a normal neigbourhood $U_{x}$ such that the unique geodesic connecting $x$ with any later point $y \in U_{x}$ (with $y=c\left(t^{\prime}\right)$ for $t^{\prime}>t$ ) is causal. To analyse such curves we introduce an auxiliary (complete) Riemannian metric $h$ on $M$ (which always exists), with associated topological metric $d_{h}$ defined as in Footnote 28, and defining things like absolute continuity etc. A continuous curve $c: I \rightarrow M$ is causal iff (possibly after reparametrization) it is absolutely continuous and a.e. differentiable on $I$ with $\dot{c}$ causal. Moreover, for $[s, u] \in I$ the Riemannian length $L_{h}\left(c_{\mid[s, u]}\right)=\int_{s}^{u} d t \sqrt{h\left(\dot{c}_{n}(t), \dot{c}_{n}(t)\right)}$ is well defined and finite. See e.g. Theorem 2.3.2 in Chruściel [37, §2.3], and Theorem A.1 in Candela et al. [16]. Since the function $u \mapsto L_{h}\left(c_{\mid[s, u]}\right)$ is strictly increasing and hence invertible, any continuous causal curve $c$ may be parametrized by $h$-arc length. If an fd (i.e. future-directed) continuous causal curve $c:[a, b)$ is parametrized by (or proportional to) $h$-arc length, then $b=\infty$ iff $c$ is future endless [134, Lemmas 2.6 and 2.17].

34 It is the second ('converse') part of the proof of Theorem 3.2 below that does not work for causal geodesics instead of curves, since the curve $\gamma$ constructed there is not necessarily a geodesic. This goes back to the definition of domains of dependence and Cauchy surfaces in terms of causal curves rather than geodesics, and may explain Penrose's [149] choices.
} 
An endless causal curve may either be complete, i.e. have infinite length, or incomplete (finite length). In the first case it may either go off to infinity, or hover around in a compact set, which is impossible in a strongly causal space-time; hence Penrose makes this assumption. In the second case (also assuming $\gamma$ is future directed for simplicity) it may either be thought of as crashing into a singularity, or leading to the edge of an extendible space-time. If $\gamma$ is not endless but has a future endpoint $y$, then

$$
I^{-}(\gamma)=I^{-}(y) .
$$

If $(M, g)$ is strongly causal, then $I^{ \pm}(x)=I^{ \pm}(y)$ iff $x=y$. The idea, then, is that an endless (continuous) causal curve $\gamma$ corresponds to an ideal point $y$ of space-time, which is not contained in $M$ but is still defined by $I^{-}(\gamma)$, this time without (3.1). By the above case distinction, at least in strongly causal space-times ideal points may be either points at infinity, or singularities, or boundary points, respectively. ${ }^{35}$

Now, if $\gamma$ does have a future endpoint $y \neq x$, the crucial condition $I^{-}(\gamma) \subset I^{-}(x)$ occurring in Definition 3.1 below-albeit in the endless case-is evidently equivalent to $I^{-}(y) \subset I^{-}(x)$, i.e. $y \ll x$, which states that there exists an fd timelike curve or signal from $y$ to $x$. If $\gamma$ is endless, on the other hand, there is no such point $y$, but we may still interpret (3.3) below as saying that timelike signals emanating from the ideal point $y$ defined by $\gamma$ (such as a singularity), or from arbitrarily nearby points, can reach $x$. This exegesis also applies to the condition $I^{-}(\gamma) \subset J^{-}(x)$, in which case some causal curve from $y$ reaches $x$.

The following definition then captures the two notions of cosmic censorship in Penrose [149]. ${ }^{36}$ We recall that these definitions and the ensuing theorem presuppose that $(M, g)$ is strongly causal. ${ }^{37}$

\footnotetext{
35 Geroch et al. [90] and in their wake Hawking and Ellis [98, §6.8], show that (assuming strong causality) both real points and ideal points of $M$ correspond to subsets $U \subset M$ that are: (i) open, (ii), past sets, i.e. $I^{-}(U) \subset U$, and (iii) indecomposable, in that $U \neq U_{1} \cup U_{2}$ where $U_{1}$ and $U_{2}$ have properties (i) and (ii) and are neither empty nor equal to $U$. Such sets are called IP (for Indecomposable Past set), and those that are not of the form $U=I^{-}(x)$ for some $x \in M$ are called TIP's (for Terminal IP's); these TIP's are $U=I^{-}(\gamma)$ for some future-endless timelike curve $\gamma$. It would be more natural if strong cosmic censorship merely excluded visible TIP's coming from incomplete curves, but Penrose [149] gives various arguments for including complete curves $\gamma$, too, and in any case his Theorem 3.2 holds only if all TIP's are included.

${ }^{36}$ See also Królak [119] for a different axiomatization of weak cosmic censorship à la Penrose, as well as useful analysis.

${ }^{37}$ Strong causality is used through its implication that $I^{ \pm}(x)=I^{ \pm}(y)$ iff $x=y$, without which Definition 3.1 would make little sense, as well as through its implication of non-total imprisonment, without which the invocation of Theorem 2.53 in Minguzzi [135] in the proof of Theorem 3.2 below would fail. It is also part of one of the traditional definitions of global hyperbolicity (namely strong causality plus compactness of causal diamonds $\left.J^{+}(y) \cap J^{-}(x)\right)$, but since the proof of Theorem 3.2 is based on contradicting compactness of causal diamonds, in that role it is hardly necessary anymore, since Hounnonkpe and Minguzzi [104] proved that a non-compact space-time with $\operatorname{dim}(M) \geq 3$ is globally hyperbolic iff all double cones are compact.
} 
Definition 3.1 In both cases below, let $\gamma$ denote a future-directed future-endless causal curve. $^{38}$

- A space-time $(M, g)$ that is asymptotically flat at null infinity (Sect. 2.1) contains a naked singularity if there is a curve $\gamma$ as above, and a point $x \in J^{-}\left(\mathscr{I}^{+}\right)$in its causal future in $\tilde{M}$, in the sense that

$$
I^{-}(\gamma) \subset J^{-}(x) .
$$

Penrose's weak cosmic censorship conjecture states that space-times that are asymptotically flat at null infinity and arise from "generic" regular initial conditions contain no naked singularities.

- A space-time $(M, g)$ contains a locally naked singularity if there is a curve $\gamma$ as above, and a point $x \in M$ in its chronological future, in the sense that

$$
I^{-}(\gamma) \subset I^{-}(x) .
$$

Penrose's strong cosmic censorship conjecture states that "generic" [in his own words: "physcially reasonable"] space-times do not contain locally naked singularities.

It should be defined precisely what "generic" means, lest these conjectures turn into a definition of genericity! Penrose did not do this, and we will return to this point in Sect. 3.2. It is important to realize that in this definition Penrose does not require $\gamma$ to be incomplete, but merely endless. Indeed the notion of (in)completeness is hard to define for non-geodesic curves since it depends on the parametrization; if, as we do, continuous causal curves are parametrized by arc length as measured by an auxiliary complete Riemannian metric (see Footnote 33), then the distinction between endlessness and incompleteness cannot even be made, because any endless curve has infinite arc length. ${ }^{39}$ Beyond moving from causal geodesics to general causal curves, this further generalization allows even more singularities, and has the effect of making the notion(s) of cosmic censorship more stringent-in excluding a larger class of naked or locally naked singularities-than Penrose's [144] singularity theorem would suggest. ${ }^{40}$

\footnotetext{
38 There is a similar definition in terms of past-directed endless causal curves, in which $I^{-}(\cdot)$ is replaced by $I^{+}(\cdot)$ throughout. As far as strong cosmic censorship is concerned this definition turns out to be equivalent to the given one, cf. Theorem 3.2, whilst for weak cosmic censorship the above definition is the appropriate one. Note that strong cosmic censorship does not imply weak cosmic censorship since (3.2) has $J^{-}(x)$ with $x$ possibly in $\mathscr{I}^{+} \subset \partial \tilde{M}$, whilst (3.3) has $I^{-}(x)$ with $x \in M$.

${ }^{39}$ Recall that affinely parametrized geodesics are incomplete iff they are endless and have finite parameter length.

${ }^{40}$ The conditions (3.2) and (3.3) do make sense for complete future endless causal curves: for example, in anti-de Sitter space one has endless causal curves $\gamma$ and points $x$ such that $I^{-}(\gamma) \subset J^{-}(x)$, but this space is not regarded as singular (it is a space-time of constant negative curvature). Penrose [149, p. 623], notes that this is impossible in space-times that are asymptotically flat at null infinity, and indeed anti-de Sitter space has a negative cosmological constant with timelike future null infinity.
} 
The following theorem, of which part 2 is due to Penrose [149] with a slightly different proof, and part 1 is an almost trivial addition, is the main characterization of cosmic censorship in his sense.

\section{Theorem 3.2}

1. If $(M, g)$ is asymptotically flat at null infinity, then it has no naked singularities iff the exterior region $J^{-}\left(\mathscr{I}^{+}\right)$in $\tilde{M}$ (which by definition includes $\mathscr{I}^{+} \subset \partial \tilde{M}$ ) is globally hyperbolic. ${ }^{41}$

2. In general, a space-time $(M, g)$ has no locally naked singularities iff it is globally hyperbolic.

It should be clear intuitively that at least part 2 of the theorem is true (in the contrapositive): if a space-time contains a locally naked singularity, represented by $\gamma$ as in Definition 3.1, then $\gamma$ will not reach any partial Cauchy-surface $\Sigma$ lying in the future of $x$, since it crashes at the singularity lying in the past of $x$. Conversely, if no Cauchy surface exists then one can construct such a curve $\gamma$. See also Sect. 4 .

Proof We prove the inference from a locally naked singularity to non-global hyperbolicity by contradiction. Suppose that (3.3) holds for some $\gamma$ and $x$ and that $(M, g)$ is globally hyperbolic. Take $y \in \gamma$ and then a future-directed sequence $\left(y_{n}\right)$ of points on $\gamma$, with $y_{0}=y$. Because of (3.3) this sequence lies in $J^{+}(y) \cap J^{-}(x)$, which is compact by assumption. Hence $\left(y_{n}\right)$ has a limit point $z$ in $J^{+}(y) \cap J^{-}(x)$. Now define curves $\left(c_{n}\right)$ as the segments of $\gamma$ from $y$ to $y_{n}$. By the curve limit lemma, ${ }^{42}$ these curves have a uniform limit, whose arc length (as measured by an auxiliary complete Riemannian metric, see Footnote 33) is on the one hand infinite (since $\gamma$ is endless and hence has infinite arc length, which is approached as the $y_{n}$ move up along $\gamma$ ), but on the other hand is finite, since it must end at $z$ (and fd continuous causal curves have finite arc length iff they have an endpoint). Hence $(M, g)$ cannot be globally hyperbolic. ${ }^{43}$

\footnotetext{
${ }^{41}$ Tipler et al. [176, p. 176], made this the definition of weak cosmic censorship. It may be closer to Penrose's [149] formulation to require $x \in J^{-}\left(\mathscr{I}^{+}\right) \cap J^{+}(\Sigma)$ in the first part of Definition 3.1, where $\Sigma$ is some partial Cauchy surface in $M$, in which case Theorem 3.2 yields global hyperbolicity of $J^{-}\left(\mathscr{I}^{+}\right) \cap J^{+}(\Sigma)$. This is similar to the condition $\mathscr{I}^{+} \subset \overline{D^{+}(\Sigma)}$ making $(M, g)$ future asymptotically predictable from $\Sigma$ [98, p. 312], but is equivalent to it only under further regularity assumptions [119, Lemma 2.10]. See also Wald [179, §12.1] and Chruściel [39, §3.5.1]

${ }^{42}$ One needs Theorem 2.53 in Minguzzi [135], of which part (i) applies: Let $\left(c_{n}:\left[0, b_{n}\right] \rightarrow M\right)$ be a sequence of fd continuous causal curves parametrized by $h$-arc length in a non-imprisoning space-time such that $c_{n}(0) \rightarrow x$ and $c_{n}\left(b_{n}\right) \rightarrow y \neq x$. Then there exists an fd continuous causal curve $c:[0, b] \rightarrow M$, where $b<\infty$ as well as a subsequence of $\left(c_{n}\right)$ that converges uniformly to $c$ (including $b_{n} \rightarrow b$ at the endpoint). Penrose [149] gives a more complicated argument, perhaps since the version of the curve limit lemma just cited was not available at the time, or because he wanted to use his TIP's (which we avoid).

43 This also works for part 1, where $x \in J^{-}\left(\mathscr{I}^{+}\right)$), Eq. (3.2) also implies $y_{n} \in J^{+}(y) \cap J^{-}(x)$. Conversely, if there are $x, y$ for which $J^{-}(x) \cap J^{+}(y)$ is not compact, one can easily construct a future-directed futureendless causal curve $\gamma$ such that (3.3) holds for the given $x$ [149, p. 624]. Since (3.3) trivially gives $I^{-}(\gamma) \subset J^{-}(x)$, also this implication works for part 1.
} 
Especially its definition through the existence of a Cauchy surface relates global hyperbolicity to determinism, the idea being that any event in a globally hyperbolic space-time is determined by certain initial data on a Cauchy surface in it, at least as long as the (classical) universe is governed by hyperbolic partial differential equations. This is clearly true for the gravitational field itself (as long as it satisfies the Einstein equations), and also has considerable backing for other fields. ${ }^{44}$ This does not imply that non-globally hyperbolic space-times are necessarily indeterministic: the point is rather that signals from a (locally) naked singularity can reach an event without ultimately coming from a Cauchy surface, so that the event is influenced by data other than those at an initial-value surface. Thus the event in question may still be fully determined-but it is not determined by the initial data that were supposed to do so. ${ }^{45}$

Conversely, the flagrant indeterminism concerning the unknown fate of someone falling into a black hole singularity is compatible with global hyperbolicity (as in e.g. the Schwarzschild solution). Furthermore, suppose some globally hyperbolic space-time $(M, g)$ solving the Einstein equations is metrically extendible (see Sect. 2.3), such that the extension $\left(M^{\prime}, g^{\prime}\right)$ is either not globally hyperbolic at all, or is globally hyperbolic but not with respect to any Cauchy surface $\Sigma$ in $M$. Then again, although all things in $\left(M^{\prime}, g^{\prime}\right)$ may be determined, they are not determined by the initial data on $\Sigma$ one expected to do so. ${ }^{46}$

\subsection{Cosmic Censorship in the Initial Value (PDE) Formulation}

Definition 3.1 of the cosmic censorship conjectures is inappropriate from the point of view of the initial-value problem. Recall from Sect. 1 that in this approach all valid questions are about the maximal globally hyperbolic development $(M, g, l)$ of initial data $(\Sigma, h, K)$. Since the MGHD is always globally hyperbolic, the strong version is trivial by Theorem 3.2. For weak cosmic censorship there is a subtle issue about which asymptotically flat initial data lead to MGHD that are asymptotically flat at null infinity (see Footnote 16), but even granting this, the real problem is that even in clear counterexamples to the Penrosian conjecture (such as $m<0$ Schwarzschild, see Sect. 4$)$, the space $J^{-}\left(\mathscr{I}^{+}\right)$computed for the MGHD is globally hyperbolic. Thus the Penrosian version, applied to the MGHD, would hold despite naked singularities!

There is no crystal-clear logical path from Penrose's formulation of the cosmic censorship conjectures to the current versions used in the PDE literature, but there is some continuity of ideas. First, as to the weak version, in order to strengthen Definition 2.1 (or rather some slight variation thereof) Geroch and Horowitz [89] proposed

\footnotetext{
${ }_{44}$ See e.g. Choquet-Bruhat [26] and Bär et al. [10], respectively, as well as Earman [66, 70].

45 The closest analogue to this generally relativistic situation occurs in non-relativistic mechanics, where bodies may disappear to infinity in finite time [164, 186], and hence, by the same (time-reversed) token, may appear from nowhere in finite time and hence influence affairs in a way unforeseeable from any Cauchy surface. See Earman [70, §3.6].

46 Doboszewski [63-65] analyzes the connection between global hyperbolicity, extendibility, and determinism.
} 
that future null infinity $\mathscr{I}^{+}$be null geodesically complete. ${ }^{47}$ This was motivated by the following example. In light-cone coordinates, the standard conformal completion $(\tilde{\mathbb{M}}, \tilde{\eta})$ of Minkowski space-time $(\mathbb{M}, \eta)$, described for example in Penrose [146, pp. 175-177], is given by

$$
\begin{gathered}
\hat{\mathbb{M}}:=\left\{(p, q, \theta, \varphi) \mid(p, q) \in\left(-\frac{1}{2} \pi, \frac{1}{2} \pi\right)^{2}, p \geq q,(\theta, \varphi) \in S^{2}\right\} \cup \mathscr{I}^{+} \cup \mathscr{I}^{-} \\
\mathscr{I}^{+}=\left\{(p, q, \theta, \varphi) \mid p=\frac{1}{2} \pi, q \in\left(-\frac{1}{2} \pi, \frac{1}{2} \pi\right),(\theta, \varphi) \in S^{2}\right\} \\
\mathscr{I}^{-}=\left\{(p, q, \theta, \varphi) \mid p \in\left(-\frac{1}{2} \pi, \frac{1}{2} \pi\right), q=-\frac{1}{2} \pi,(\theta, \varphi) \in S^{2}\right\} \\
\hat{\eta}=-d p d q+\frac{1}{4} \sin ^{2}(p-q)\left(d \theta^{2}+\sin ^{2} \theta d \varphi^{2}\right) \\
\Omega=\cos p \cos q .
\end{gathered}
$$

Now on the one hand, truncating $\mathscr{I}^{+}$to for example $\left\{(p, q, \theta, \varphi) \mid p=\frac{1}{2} \pi, q \in\left(-\frac{1}{2} \pi, 0\right)\right\}$ instead of (3.5) would still define a conformal completion of $(\mathbb{M}, \eta)$, with respect to which the future light-cone $J^{+}(0)$ is a fake black hole $\mathscr{B}$ in $\mathbb{M}$. On the other hand, removing $\mathscr{B}=J^{+}(0)$, the ensuing space-time $\left(\mathbb{M} \backslash J^{+}(0), \eta\right)$ has a conformal completion (such as the one just described), which by design is free of black holes. In both undesirable cases future null infinity is incomplete (in the sense of Footnote 47). ${ }^{48}$

Completeness of future null infinity in the above sense, then, was taken to be the PDE reformulation of weak cosmic censorship [32], although it turns the Penrosian version on its head! For whereas his version states that outgoing signals from a black hole singularity are blocked by an event horizon $\mathscr{H}_{E}^{+}$, the new version is about incoming (null) signals: the further these are away from $\mathscr{H}_{E}^{+}$, the longer it takes them to enter $\mathscr{H}_{E}^{+}$, and in the limit at null infinity this takes infinitely long, making $\mathscr{I}^{+}$

\footnotetext{
47 This condition is nontrivial to state; for example, in the metric $\tilde{\eta}$ used below even $\mathscr{I}^{+}$for the standard conformal completion $(\tilde{\mathbb{M}}, \tilde{\eta})$ of Minkowski space-time $(\mathbb{M}, \eta)$ is incomplete. Completeness of curves depends on their parametrization. Geodesics are affinely parametrized by definition (and an affine reparametrization does not affect their (in)completeness), but a change in $\Omega$ changes the unphysical metric $\tilde{g}$ (for given physical metric $g$ ). Hence the notion of a geodesic and its (in)completeness depends on the choice of $\Omega$. As recognized by Geroch and Horowitz [89] themselves, the correct approach is to use the freedom of rescaling $\Omega$ to ensure that $\tilde{\nabla}_{\mu} \tilde{\nabla}_{\nu} \Omega=0$ on $\mathscr{I}^{+}$, and require null geodesic completeness of the null hypersurface $\mathscr{I}^{+}$in this "gauge", in which the flow of $\tilde{\nabla} \Omega$ is geodesic. See also Wald [179, §11.1], or Stewart $[174, \S 3.6]$.

${ }^{48}$ This clause seems to be an improvement over an inextendibility condition proposed by Geroch [88], which did not exclude cases like $\left(\mathbb{M} \backslash J^{+}(0), \eta\right)$. However, inextendibility plus some regularity condition enabled Geroch [88] to prove uniqueness of conformal completions, a result that seems to have no analogue for Definition 2.1, even in strengthened form.
} 
complete. Yet the PDE version appears to strengthens Penrose's: heuristically, and contrapositively, lack of global hyperbolicity of $J^{-}\left(\mathscr{I}^{+}\right)$gives a partial Cauchy surface $\Sigma$ a Cauchy horizon which cuts off $\mathscr{I}^{+}$, making it incomplete (this will perhaps be clearer from the examples in Sect. 4). ${ }^{49}$ Thus we obtain:

\section{Definition 3.3}

- The weak cosmic censorship conjecture states that if "generic" complete initial data have a MGHD that is asymptotically flat at null infinity, then future null infinity is complete.

- The strong cosmic censorship conjecture states that the MGHD of "generic" complete initial data is metrically inextendible (as a space-time in a regularity class to be specified in detail).

For convenience, we have added the strong version of cosmic censorship used in the PDE approach, whose path from the Penrosian formulation we now try to trace. ${ }^{50}$ First, in a paper on weak cosmic censorship, Moncrief and Eardley [138, p. 889], propose an '(informally stated) global existence conjecture':

Every asymptotically flat initial data set with $\operatorname{tr} K=0$ may be evolved to arbitrarily large times (...)

adding that its proof would 'in essence prove the [weak] cosmic censorship conjecture for asymptotically flat space-times'. For initial data given on a compact Cauchy surface they propose something similar, and in doing so they opened the door to regarding cosmic censorship as a global existence problem for the (vacuum) Einstein equations, as indeed the title of their paper already expresses. In this spirit, Moncrief [137, p. 88], paraphrases Penrose's strong version as expressed by Theorem 3.2 as

i.e., that the maximal Cauchy development of a generic initial data set is inextendible.

This is made more precise by Chruściel et al. [43], who open their abstract as follows:

\footnotetext{
${ }^{49}$ Christodoulou [32] actually reformulates the above definition of weak cosmic censorship in such a way that the idealization $\mathscr{I}^{+}$no longer occurs. Let $(\Sigma, h, K)$ be asymptotically flat initial data for the Einstein equations (satisfying the constraints), with MGHD $(M, g, i)$. He then defines $(M, g)$ to have "complete future null infinity" iff for any $s>0$ there exists a region $B_{0} \subset B \subset \Sigma$ such that $\partial D^{+}(B)$, which is ruled by null geodesics, has the property that each null geodesic starting in $\partial J^{+}\left(B_{0}\right) \cap \partial D^{+}(B)$ can be future extended beyond parameter value $s$. Here $D^{+}(B)$ is the future domain of dependence of $B$, and each null geodesic in question is supposed to have tangent vector $L=T-N$, where $T$ is the fd unit normal to $\Sigma$ in $M$ and $N$ is the outward unit normal to $\partial B$ in $\Sigma$. See also Christodoulou and Klainerman [35] for background on these constructions.

${ }^{50}$ I am greatly indebted to Juliusz Doboszewski for drawing my attention to the early papers by Moncrief et al.
} 
The strong cosmic censorship conjecture states that 'most' spacetimes developed as solutions of Einstein's equations from prescribed initial data cannot be extended outside of their maximal domains of dependence.

They later (§3) specify the word 'most' in terms of open and dense subsets in the space of initial data. ${ }^{51}$

Chruściel [36] introduced the notion of a development of initial data $(\Sigma, h, K)$ as a triple $(M, g, i)$, where $(M, g)$ is a $4 d$ space-time solving the vacuum Einstein equations, and $i: \Sigma \rightarrow M$ is an embedding such that $i^{*} g=h$ and $i(\Sigma)$ has extrinsic curvature $K$; the difference from a Cauchy development (see Footnote 12 ) is that $i(\Sigma)$ is no longer required to be Cauchy surface in $M$, so that $(M, g)$ is not necessarily globally hyperbolic. He calls such a development maximal if there is no extension $\left(M^{\prime}, g^{\prime}\right)$ that also satisfies the vacuum Einstein equations, and proves existence of maximal developments (but not uniqueness up to isometry, as in the globally hyperbolic case, cf. Footnote 12). Applying Penrose's strong cosmic censorship to such a maximal development, he asks it to be globally hyperbolic. If this is the case, then-up to isometry as usual- $(M, g)$ must coincide with the MGHD of given initial data. ${ }^{52}$ Consequently, this specific application of strong cosmic censorship à la Penrose is equivalent to asking the MGHD of given initial data to be inextendible as a solution to the vacuum (or any kind of) Einstein equations. ${ }^{53}$

Adding suitable regularity conditions on the extensions, ${ }^{54}$ this would be a meaningful and natural PDE version of strong cosmic censorship but the version used in the PDE literature is stronger: one requires metric inextendibility of the MGHD full stop, whether or not this extension satisfies the vacuum Einstein equations. And although it would make sense in general, in practice the ensuing conjecture is posed for either non-compact $\Sigma$ with asymptotically flat initial data or compact $\Sigma$; better safe than sorry!

The need for a restriction on the scope of the conjectures was clearly realized and stated-albeit purely qualitatively_already by Penrose himself (see Footnote 11). Indeed, without such a restriction some of the best-known exact black hole solutions (cf. Sect. 4) provide counterexamples to one or both of the conjectures, as was of course well known to Penrose and his circle (for the Penrosian version, that is). To get around this, in one of his most prophetic insights, Penrose (1968, p. 222)

\footnotetext{
51 Detailed mathematical criteria for genericity (which are suggested by PDE theory and whose physical relevance is doubted outside the PDE community) may be also be found for example in Dafermos [51] and Luk and Oh [127, §3].

52 Continuing Footnote 12 , the set of isometry classes $[M, g, i]$ of Cauchy developments $(M, g, i)$ of given initial data $(\Sigma, h, K)$ is partially ordered by $\left[M_{1}, g_{1}, i_{1}\right] \leq\left[M_{2}, g_{2}, i_{2}\right]$ provided there are representatives $\left(M_{1}^{\prime}, g_{1}^{\prime}, i_{1}^{\prime}\right)$ and $\left(M_{2}^{\prime}, g_{2}^{\prime}, i_{2}^{\prime}\right)$ and an embedding $\psi: M_{1}^{\prime} \rightarrow M_{2}^{\prime}$ for which $\psi^{*} g_{2}^{\prime}=g_{1}^{\prime}$ and $\psi \circ l_{1}^{\prime}=l_{2}^{\prime}$. The MGHD $\left[M_{t}, g_{t}, i_{t}\right]$ is the top element of this poset [166] and so if some maximal development $\left(M_{m}, g_{m}, i_{m}\right)$ à la Chruściel is globally hyperbolic then $\left[M_{m}, g_{m}, i_{m}\right] \leq\left[M_{t}, g_{t}, i_{t}\right]$. On the other hand, since $\left(M_{t}, g_{t}, i_{t}\right)$ is a solution and $\left(M_{m}, g_{m}, i_{m}\right)$ is maximal also the converse holds, so $\left(M_{m}, g_{m}, i_{m}\right) \cong\left(M_{t}, g_{t}, i_{t}\right)$.

53 See Doboszewski [63, 64] and Manchak [131, 132] for conceptual studies of the (in)extendibility of space-times.

54 As pointed out to me by Julius Doboszewski, Chruściel et al. [43] as well as Chruściel and Isenberg [42] only consider smooth extensions, so that looking at lower regularity seems a refinement postdating this early phase.
} 
suggested that because of a blueshift instability of the Cauchy horizon under perturbations, it turns into a curvature singularity:

Our contention in this note is that if the initial data is generically perturbed then the Cauchy horizon does not survive as a non-singular hypersurface. It is strongly implied that instead, genuine space-time singularities will appear along the region which would otherwise have been the Cauchy horizon. [172, p. 184]

Since then, this instability has been confirmed in a large number of studies, starting with Hiscock [102] in the physics literature and Dafermos [51] in the mathematical one; recent papers include Chesler et al. [24] and Van de Moortel [178], respectively. The conclusion seems to be that Cauchy horizons turn into so-called weak null singularities, ${ }^{55}$ behind which-at least for one-ended asymptotically flat initial data-there is a strong curvature singularity at $r=0$. See also Luk and Oh [127, 128] for the two-ended case. Unappealingly, the sense in which strong cosmic censorship (in the PDE formulation) then fails or holds depends critically on the regularity assumptions of the extension. ${ }^{56}$

For example, for two-ended asymptotically flat data for the spherically symmetric Einstein-Maxwell-scalar field system (to which the conjecture, so far discussed for the vacuum case, can be extended in the obvious way), the strong cosmic censorship conjecture fails in $C^{0}[60],{ }^{57}$ but it holds in $C^{0}$ with the additional requirement that the associated Christoffel symbols are locally $L^{2}[127,128]$. This is not just a technicality, since having the metric in $C^{0}$ and its Christoffel symbols locally $L^{2}$ is a borderline regularity condition for metric extensions in strong cosmic censorship: it is the least regular case in which the metric can still be defined as a weak solution to Einstein's equations [34, p. 9], [126, Footnote 1]. Indeed, a weak solution of the vacuum Einstein equations is a metric $g$ for which for all compactly supported $X, Y \in \mathfrak{X}(M)$,

$$
\int_{M} d^{4} x \sqrt{-\operatorname{det}(g(x))} R_{\mu \nu}(x) X^{\mu}(x) Y^{\nu}(x)=0 .
$$

Partial integration shows that this is well defined iff the $\Gamma_{\mu \nu}^{\rho}$ are locally $L^{2}$. This simple observation should not be confused with the very deep result that having the Ricci tensor in $L^{2}$ is sufficient for the (vacuum) Einstein equations to be weakly solvable at least locally [117]. Ironically, in Definition 3.3 of strong cosmic censorship the extension is not required to satisfy the Einstein (or indeed any other) equations!

\footnotetext{
$\overline{55}$ These are null boundaries with $C^{0}$ metric but Christoffel symbols not locally in $L^{2}[126,129]$.

56 The results below concern cosmological constant $\lambda=0$ and subextremal black holes (i.e. $e^{2}<m^{2}$ for $\mathrm{R}-\mathrm{N}$ and $a^{2}<m^{2}$ for Kerr). See Dias et al. [62] for $\lambda>0$ : strong cosmic censorship seems true in pure gravity and false for the Einstein-Maxwell system, but again this depends critically on the regularity of the extension. For extremal Reissner-Nordström $\left(e^{2}=m^{2}\right)$ at $\lambda=0$ see Gajic and Luk [85], suggesting failure of strong cosmic censorship, as is trivially the case for $e^{2}>m^{2}$.

57 Their general result assumes the (widely expected) stability of the Kerr metric under perturbations of the initial data.
} 
See also Ringström [156] for a review of the 'cosmological' case where the Cauchy surface is compact, in which the strong (PDE) conjecture seems to hold.

The status of weak cosmic censorship is even less clear. Christodoulou [33] proves the conjecture for the spherically symmetric gravitational collapse of a scalar field, but on the basis of genericity conditions whose relevance has been questioned in the physics literature [94, §3.4]. More generally, the status of weak cosmic censorship seems mixed also in earlier heuristic formulations in terms of an event horizon; see e.g. Joshi [110, 111], Królak [120, 121], and Ong [140].

\section{Examples}

The relationship between the Penrosian and the PDE versions of the cosmic censorship conjectures is best understood from three key black hole examples and their Penrose diagrams: ${ }^{58}$

- Maximally extended Schwarzschild (i.e. Kruskal) with $m>0$ (and two-sided initial data);

- Schwarzschild with $m<0$, which in so far as singularities and horizons are concerned also looks like supercharged Reissner-Nordström $\left(e^{2}>m^{2}>0\right)$, or fast rotating $\operatorname{Kerr}\left(a^{2}>m^{2}>0\right)$;

- Reissner-Nordström with $0<e^{2}<m^{2}$, which qualitatively also represents Kerr with $0<a^{2}<m^{2}$.

In the first case the solution coincides with the MGHD of the corresponding (twoended) initial data, so the difference between the Penrosian and the PDE approach evaporates. Here is the Penrose diagram:

\footnotetext{
${ }^{58}$ Even more so than the previous sections this one is purely pedagogical and drawn largely from Hawking and Ellis [98, pp. 158 and 160], as well as from Dafermos and Rodnianski [61] and Dafermos [5357] for the PDE side. 


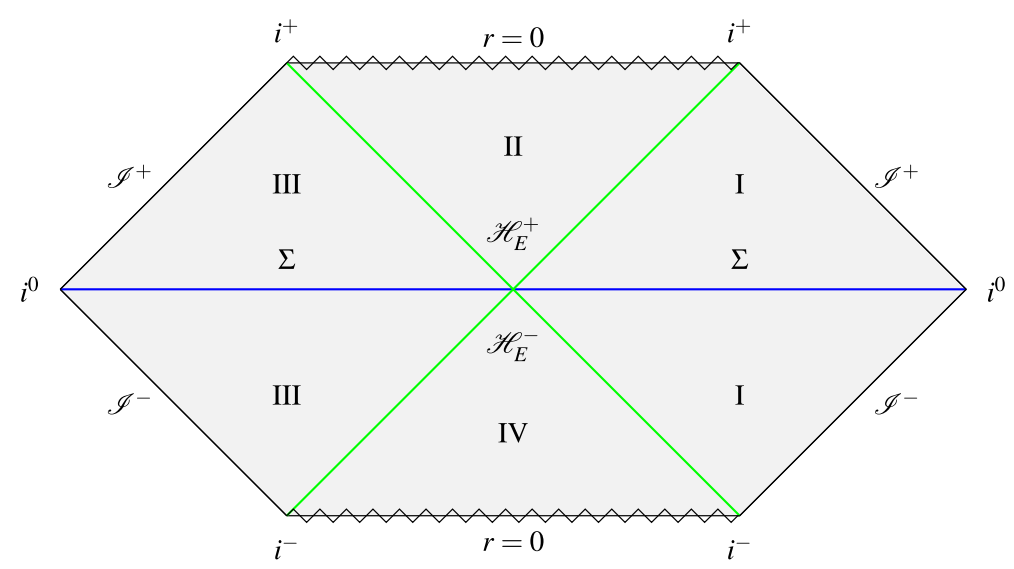

Penrose diagram of the maximally extended Schwarzschild solution with $m>0$. This solution coincides with the maximal Cauchy development (marked in light grey) of a generic two-sided Cauchy surface $\Sigma$ with suitable initial data, drawn as a horizontal blue line. Thus the Cauchy horizon $\mathscr{H}_{C}^{ \pm}$is empty. The upper two green lines form the future event horizon $\mathscr{H}_{E}^{+}$of the black hole area, which is the upsidedown upper triangle (labeled region II), whereas the lower two green lines form the past event horizon $\mathscr{H}_{E}^{-}$of the white hole area, i.e. the lower triangle (region IV). The right-hand diamond is region I, the left-hand diamond is region III. Fd causal curves cannot leave region II and they cannot enter region IV.

Both cosmic censorship conjectures hold in both versions (i.e. Penrose and PDE):

- Weak cosmic censorship. Penrose: $\Sigma$ is a Cauchy surface for $J^{-}\left(\mathscr{I}^{+}\right)$, making it globally hyperbolic. ${ }^{59}$ PDE: each component of $\mathscr{I}^{+}$ends at timelike infinity and hence all its null geodesics are future complete (as confirmed by explicit parametrization and computation).

- Strong cosmic censorship. Penrose: Kruskal space-time is globally hyperbolic (since the causal structure of the diagram is such that the line $\Sigma$ represents a Cauchy surface). PDE: For smooth extensions Remark 5.45 on p. 155 of O'Neill [139] or Proposition 4.4.3 in Chruściel [39] plus a detailed study of the geodesics shows that Kruskal space-time is metrically inextendible. ${ }^{60}$

\footnotetext{
59 Alternatively: any incomplete future inextendible timelike curve $\gamma$ must crash in the upper $r=0$ singularity. Hence $I^{-}(\gamma)$ lies partly in region II, which is disjoint from $J^{-}\left(\mathscr{I}^{+}\right)$, so that $I^{-}(\gamma) \nsubseteq J^{-}(x)$ for all $x \in J^{-}\left(\mathscr{I}^{+}\right)$.

${ }^{60}$ If for any maximally extended timelike geodesic $\gamma:[0, b) \rightarrow M$ in $M$ there is a curvature invariant (such as $R$ or $R^{\rho \sigma \mu \nu} R_{\rho \sigma \mu \nu}$, etc.) that blows up as $\gamma(t) \rightarrow b$, then $(M, g)$ is inextendible. See O'Neill [139, Chap. 13], for a study of Kruskal geodesics, proving the antecedent. Sbierski [167, 168] proves that Kruskal space-time is inextendible even in $C^{0}$.
} 
However, for $m<0$ Kruskal, Reissner-Nordström, and Kerr, differences arise between the Penrosian and the PDE perspectives, since in these cases the maximal (analytic) solutions, deemed unphysical by the PDE aficionados, differ from the MGHD of the pertinent initial data. In particular, although (curvature) singularities are not part of space-time in any case, they can at least be drawn as boundaries in the maximal solutions, where they lie behind a Cauchy horizon. But precisely for that reason singularities are outside any kind of scope of the corresponding MGHD. Here are the Penrose diagrams:
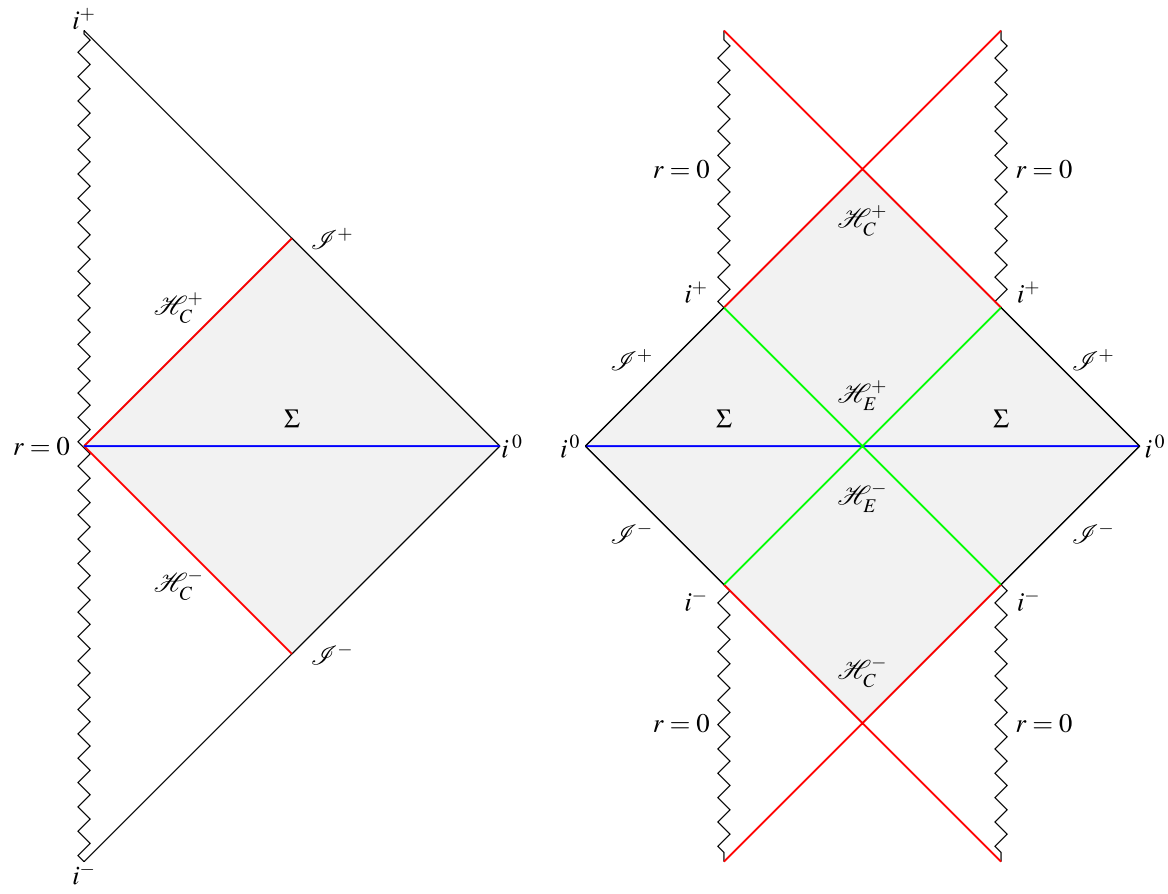

Left picture: Penrose diagram of $m<0$ Schwarzschild, or supercharged Reissner-Nordström $\left(e^{2}>m^{2}>0\right)$, or fast Kerr $\left(a^{2}>m^{2}>0\right)$. These solutions have a singularity at $r=0$, but unlike the $m>0$ Kruskal case it is not shielded by an event horizon. Instead, the red lines labeled $\mathscr{H}_{E}^{-}$and $\mathscr{H}_{E}^{+}$are past and future Cauchy horizons with respect to the blue line, indicating a maximal spacelike surface whose initial data give rise to the metrics in question and whose maximal Cauchy development is the grey area.

Right picture: Penrose diagram of subcritical Reissner-Nordström $\left(0<e^{2}<m^{2}\right)$, whose event and Cauchy horizons (despite the different structure of the singularity) also resemble those of slowly rotating $\operatorname{Kerr}\left(0<a^{2}<m^{2}\right)$. The maximal Cauchy development of the pertinent initial data given on the maximal spacelike hypersurface represented by the blue line labeled $\Sigma$ is again colored in grey. It contains past and future event horizons labeled $\mathscr{H}_{E}^{-}$and $\mathscr{H}_{E}^{+}$, drawn in green, but unlike the $m>0$ Schwarzschild case the singularity they are supposed to shield cannot be reached 
directly from the maximal Cauchy development, which is bounded by the various fictitious boundaries $\mathscr{J}^{ \pm}, i^{ \pm}$, and $i^{0}$, which lie at infinity, as well as by the Cauchy horizons $\mathscr{H}_{C}^{ \pm}$, drawn in red, which can be reached in finite proper time. ${ }^{61}$

Despite the different space-times they apply to, the outcomes of the Penrosian version and the PDE version of both weak and strong cosmic censorship are once again the same, mutatis mutandis: ${ }^{62}$

- $m<0$ Kruskal (etc.): for the Penrosian total space-time the difference between weak and strong cosmic censorship fades since $J^{-1}\left(\mathscr{I}^{+}\right)=M \cup \mathscr{I}^{+}$, which, like $M$ itself is not globally hyperbolic: wherever one tries to place a partial Cauchy surface $\Sigma$ (such as the blue line), above the surface inextendible causal curves can be drawn that enter $i^{+}$or $\mathscr{I}^{+}$in the future and enter the singularity at $r=0$ in the past, without crossing $\Sigma$. Similarly, below $\Sigma$ one may draw inextendible causal curves converging to the singularity in the future, and to $i^{-}$or $\mathscr{I}^{-}$in the past, which once again do not cross $\Sigma$. Thus neither weak nor strong cosmic censorship holds for this space-time.

The PDE picture applies to the grey area, which is the MGHD of the initial data given on the blue line marked $\Sigma$ in the left-hand Penrose diagram. Then weak cosmic censorship fails because future null infinity $\mathscr{I}^{+}$is clearly incomplete: null geodesics terminate at the Cauchy horizon (where they "fall off' space-time) and hence are incomplete. On the other hand, strong cosmic censorship fails because the grey space-time, though globally hyperbolic (in contrast with the entire space as we have just seen), is evidently (smoothly - even analytically) extendible, namely by the total space. Though they do not coincide, we see that strong and weak cosmic censorship are closely related: future incompleteness of null geodesics at null infinity happens because the MGHD is extendible.

- Subcritical Reissner-Nordström $\left(0<e^{2}<m^{2}\right)$ : for both Penrose and the PDE people strong cosmic censorship fails whereas the weak version holds. In the Penrosian version the total space fails to be globally hyperbolic because of the part above the grey area (i.e. beyond the future Cauchy horizon $\mathscr{H}_{E}^{+}$): one has past-directed inextendible causal curves that (backwards in time) end up in the singularity and hence never cross $\Sigma$ (e.g. those crossing the upper left, NW-pointing red line from $\mathrm{N}$ to $\mathrm{SW}$ ). Morally, weak cosmic censorship holds because of the future event horizon $\mathscr{H}_{E}^{+}$, which shields the upper $r=0$ singularity above it, but legally this is only the case if we stop the Penrose diagram at the past Cauchy horizon $\mathscr{H}_{E}^{-}$, as we have done in drawing the picture (for otherwise causal curves below it may crash at the lower $r=0$ singularity and hence never reach $\Sigma$ ).

\footnotetext{
61 This diagram can be infinitely extended in both directions [98, pp. 158, 165]: to the north, another grey area folds inside the upper two red line segments, and similarly to the south, et cetera, but we do not do so here.

${ }^{62}$ For $m<0$ Kruskal the initial data are not complete in this case, so strictly speaking the cosmic censorship conjectures do not apply here and their falsity is unimportant. Nonetheless, they can be stated and the comparison is instructive.
} 
The PDE view is cleaner here: roughly speaking, as in the $m>0$ Kruskal or Schwarzschild case (but unlike the $m<0$ case) future null infinity $\mathscr{I}^{+}$ends at future timelike infinity $i^{+}$and hence is complete, so that weak cosmic censorship holds. Strong cosmic censorship, on the other hand, fails because the MGHD (marked in grey) is clearly extendible (namely into the Penrosian space-time!).

More generally, if the strong Penrosian conjecture fails for some space-time $\left(M_{P}, g_{P}\right)$, then its lack of global hyperbolicity typically occurs because $\left(M_{P}, g_{P}\right)$ is an extension of the MGHD $(M, g)$ of some given initial data, whose Cauchy surface $\Sigma$ fails to be one for $\left(M_{P}, g_{P}\right)$. Similarly, if $J^{-1}\left(\mathscr{I}^{+}\right)$is not globally hyperbolic (so that there is a naked singularity), $M_{P}$ usually comes from extending some $(M, g)$, as above, whose Cauchy surface becomes a partial Cauchy surface in $M_{P}$, with an associated future Cauchy horizon that cuts off $\mathscr{I}^{+} \cap \tilde{M}$, causing its incompleteness. ${ }^{63}$ As already mentioned, any counterexamples are believed to be "non-generic", assuming of course that the conjectures hold!

Such reasoning, which applies to many case studies, also suggests a compromise between the Penrosian and PDE versions of cosmic censorship: informally one might say that, in "physically reasonable" space-times, weak cosmic censorship postulates the appearance and stability of event horizons, whereas strong cosmic censorship requires the instability and ensuing disappearance of Cauchy horizons.

\section{Epilogue: Penrose's Final State Conjecture}

In practice, the cosmic censorship conjectures are not put in the full generality of either Penrose's own version as expressed by Theorem 3.2 or of the PDE version as Definition 3.3, but are posed in the context of black holes as they are expected to occur in the universe, i.e. as described by something like the Kerr metric (at least outside its Cauchy horizon and more safely even outside its event horizon). As such, on the one hand they gain focus, but on the other hand they can be thought of as forming part of a broader conjecture that also originated with Penrose himself and is often called the final state conjecture ${ }^{64}$

A body, or collection of bodies, collapses down to a size comparable to its Schwarzschild radius, after which a trapped surface can be found in the region surrounding the matter. Some way outside the trapped surface region is a surface which will ultimately be the absolute event horizon. But at present, this surface is still expanding somewhat. Its exact location is a complicated affair

\footnotetext{
63 However, these aren't rigorous deductions: there are pathological cases where strong cosmic censorship holds whilst the weak version fails. See the Penrose diagram at the end of $\$ 2.6 .2$ of Dafermos and Rodnianski [61] for an example.

64 This is sometimes stated somewhat differently, in that 'generic asymptotically flat vacuum initial data (...) evolve to a solution which either disperses (in which case there are no black holes) or else eventually asymptotes to finitely many Kerr solutions (...) moving away from each other' [47, p. 78]. See also the fascinating lecture by Klainerman [113].
} 
and it depends on how much more matter (or radiation) ultimately falls in. We assume only a finite amount falls in and that GIC is true. Then the expansion of the absolute event horizon gradually slows down to stationarity. Ultimately the field settles down to becoming a Kerr solution (in the vacuum case) or a KerrNewman solution (if a nonzero net charge is trapped in the "black hole"). [147, pp. 1157-1158]

Here GIC refers to what Penrose [147] called the Generalized Israel Conjecture, ${ }^{65}$ which states that:

if an absolute event horizon develops in an asymptotically flat space-time, then the solution exterior to this horizon approaches a Kerr-Newman solution asymptotically with time. [147, pp. 1156]

In the stationary case, which is what Israel himself conjectured and proved under fairly restrictive assumptions, this would simply say that the solution exterior to this horizon equals a Kerr-Newman solution. As such, the conjecture is an outgrowth of what (following Wheeler) used to be called the "no hair" property of black holes, to the effect that stationary black holes (and eventually all black holes) are characterized by by just three parameters, viz. mass, angular momentum, and electric charge. As such, the final state conjecture incorporates not only weak cosmic censorship (notably in the compromise version suggested at the end of the previous section) but also what in the PDE literature is called Kerr stability, as well as Kerr rigidity. The former is the conjecture that generic perturbations of the initial data for the Kerr metric lead to a MGHD that is close to the original one (at least outside the event horizon). ${ }^{66}$ This would generalize the remarkable theorem on the stability of Minkowski space-time [35], which launched the modern era in PDE-oriented mathematical relativity. The latter is a more modest version of the no-hair or black hole uniqueness theorems of Israel, Carter, Hawking, Robinson, and others, ${ }^{67}$ where short of proving the stationary case of the above GIC, which requires unphysical analyticity assumptions, one tries to show that at least stationary solutions to Einstein's vacuum (electrovac) equations that are close to Kerr (-Newman) actually coincide with the latter. $^{68}$

In conclusion, "the discovery that black hole formation is a robust prediction of the general theory of relativity" still lies in the future as far as mathematical proof is concerned. Penrose's Nobel Prize was effectively awarded for a theorem and a conjecture, but it was fully deserved in every conceivable way!

\footnotetext{
$\overline{65}$ The reference is to Israel [106, 107]. See Israel [109, §7.9] and Thorne [175, Chap. 7], for interesting history.

66 There is certain numerical evidence for this (Zilhão et al. 2014), but mathematical results so far are preliminary [59, 91], except for positive cosmological constant and small $a$ [101], where the problem is solved. Even the Schwarzschild case is still open, despite impressive progress [58, 118].

67 These theorems are reviewed in Hawking and Ellis [98, \$9.3], [21, 22], Heusler [99], Robinson [160], Chruściel et al. [44], and Cederbaum [23]. See also Cardoso and Gualtieri [17] for possible tests.

68 See e.g. Alexakis et al. [3] as well as the review by Ionescu and Klainerman [105].
} 


\section{Appendix: A Potted Early History of "black hole" (by Erik Curiel)}

In reply to a query in an early draft of this paper concerning the origin of the definitions $\mathscr{B}:=M \backslash J^{-}\left(\mathscr{I}^{+}\right)$of a black hole (region) and of the event horizon as its boundary $\mathscr{H}_{E}^{+}:=\partial \mathscr{B}$, see (2.4) and (2.5), phrased as: 'Such definitions are routinely used in e.g. Carter [18] and Hawking and Ellis [98, §9.2], but who stated them first?', Erik Curiel very kindly supplied the following information. ${ }^{69}$ As we see, the question still remains somewhat open. A puzzling point is that although Penrose himself would have been the obvious person to state these definitions mathematically, apparently he did not do so!

Penrose [146] defines (p. 188), an event horizon as the boundary of the chronological past of a timelike curve (essentially the same definition, including the name, as given by [155]), and notes (p. 206) that $r=2 M$ in Schwarzschild is one. The term "black hole" does not appear in that essay, nor any definition remotely like 'the complement of the causal past of future null infinity'. Given the magisterial depth and encyclopedic scope of that essay, I must conclude that the definition was not then yet extant. Penrose [147] does use the term "black hole" (the first use of it I know in the general relativity literature, though it reportedly was used in the early 1960s by Dicke in discussion with a popular science writer), but he always encloses it in scare quotes, leading me to believe that the name and the general concept both were still inchoate. This is buttressed by the fact that he does give here (p. 1146, Footnote 3 ) the classic definition of an 'absolute event horizon' (the boundary of the chronological past of future null infinity), but he does not explicitly link it to the term "black hole". That is the first appearance of the classic definition I know of in the literature.

Ruffini and Wheeler gave a series of lectures in September 1969 at the Interlaken Colloquium on the Significance of Space Research for Fundamental Physics (Interlaken, Switzerland), one of which was entitled 'Black Holes', at least according to the expanded version of the lectures published as Ruffini and Wheeler [161], from which Ruffini and Wheeler [162] was adapted. This is the first use of the term I have been able to find recorded in a public forum in the relativity community. They explain the idea in informal, intuitive terms. Bardeen [11], received 23 January 1970, has "black hole" in the title, the first publication I know of to do so. He introduces "black hole" using scare quotes and equates it with a 'collapsed object'. Israel [108], originally read at the Gwatt Seminar on the Bearings of Topology upon General Relativity on 19 May 1970, uses "black hole" without defining it, but it is clear from context that he means something like 'system that quickly settles down so that its exterior is modeled by the Kerr solution'. Christodoulou [30], received 17 September 1970, uses the term without blushing, not even an informal gloss given

\footnotetext{
69 As in the rest of the paper, single quotation marks below denote literal quotation whereas double ones are scare quotes.
} 
for its meaning. He simply begins by talking of a 'black hole [with] angular momentum' without even citing Kerr [112]. Three papers then appear in 1971 with "black hole" in the title, Penrose and Floyd [151], received 16 December 1970, Carter [19], received 18 December 1970, and Hawking [96], received 11 March 1971. They are the only other papers from 1971 I can find related to the topic whose authors plausibly could have proposed the classic definition. Penrose and Floyd [151] uses scare-quotes around the first use of "black hole"; their discussion relies only on the event horizon and ergosphere (which they refer to as the 'stationary limit') defined by the Kerr metric, with no attempt at (or mention of the possibility of) generalization. Carter [19] does not give a formal definition of "black hole", but he does give an informal definition of 'domain of outer communication', and says (p. 331) that " "black holes" [are] regions of space-time beyond the domain of outer communication.' Note the scare-quotes. Hawking [96] uses scare-quotes as well, going out of his way to assimilate the idea of a black hole to one more widely known ('there are initially two collapsed objects or "black holes", , p. 1345). He also comes achingly close to defining a black hole as a connected component of the complement of the causal past of future null infinity, but never quite does it. He rather says things like, 'On $\Sigma_{i}$ [a spacelike hypersurface], there will be two separate regions, $B_{1}$ and $B_{2}$ which contain closed, trapped surfaces (...) Just outside $B_{1}$ and $B_{2}$ will be two two-spheres which are the intersection of $\dot{J}^{-}\left(\mathscr{I}^{+}\right)$with $\Sigma_{i}$.' The first explicit definition I know of "black hole" as "connected component of the complement of the causal past of future null infinity' is in Hawking [97]. But I am not confident that I have found all relevant sources in the literature; even if I have, one cannot be confident based only on this that Hawking was the one who finally put all the pieces together.

(Erik Curiel, private communication, January 6, 2021, reprinted with permission)

Acknowledgements This paper grew out of a seminar in honour of Roger Penrose and his Nobel Prize at Nijmegen on October 28th, 2020, at the kind invitation of Timothy Budd. The author is grateful to Carlo Rovelli for encouragement to publish it in Foundations of Physics, as well as to Béatrice Bonga, Jeremy Butterfield, Erik Curiel, Juliusz Doboszewski, John Earman, Dejan Gajic, and Dennis Lemkuhl for helpful feedback on earlier drafts. I also learned a lot form various seminars by Martin Lesourd.

Open Access This article is licensed under a Creative Commons Attribution 4.0 International License, which permits use, sharing, adaptation, distribution and reproduction in any medium or format, as long as you give appropriate credit to the original author(s) and the source, provide a link to the Creative Commons licence, and indicate if changes were made. The images or other third party material in this article are included in the article's Creative Commons licence, unless indicated otherwise in a credit line to the material. If material is not included in the article's Creative Commons licence and your intended use is not permitted by statutory regulation or exceeds the permitted use, you will need to obtain permission directly from the copyright holder. To view a copy of this licence, visit http://creativecommons.org/licen ses/by/4.0/. 


\section{References}

1. Adamo, T.M., Newman, E.T., Kozameh, C.: Null geodesic congruences, asymptotically-flat spacetimes and their physical interpretation. Living Rev. Relativ. 15(1), 6 (2012)

2. Alaee, A., Lesourd, M., Yau, S.-T.: A localized spacetime Penrose inequality and horizon detection with quasi-local mass (2019). arXiv:1912.01581. Accessed on 23 March 2021

3. Alexakis, S., Ionescu, A.D., Klainerman, S.: Rigidity of stationary black holes with small angular momentum on the horizon. Duke Math. J. 14, 2603-2615 (2014)

4. Ashtekar, A.: Asymptotic structure of the gravitational field at spatial infinity. In: Held, A. (ed.) General Relativity and Gravitation: One Hundred Years After the Birth of Albert Einstein, vol 2, pp 37-70, vol. 2. Plenum, New York (1980)

5. Ashtekar, A.: Geometry and physics at null infinity (2015). arXiv:1409.1800. Accessed on 23 March 2021

6. Ashtekar, A., Bonga, B., Kesavan, A.: Asymptotics with a positive cosmological constant: I. Basic framework. Class. Quantum Gravity 32, 025004 (2015)

7. Ashtekar, A., Galloway, G.J.: Some uniqueness results for dynamical horizons. Adv. Theor. Math. Phys. 9, 1-30 (2005)

8. Ashtekar, A., Magnon, A.: Asymptotically anti-de Sitter space-times. Class. Quantum Gravity 1, L39-L44 (1984)

9. Athanasiou, N., Lesourd, M.: Construction of Cauchy data for the dynamical formation of apparent horizons and the Penrose Inequality (2020). arXiv:2009.03704. Accessed on 23 March 2021

10. Bär, C., Ginoux, N., Pfäffle, F.: Wave Equations on Lorentzian Manifolds and Quantization. European Mathematical Society, Zurich (2007)

11. Bardeen, J.: Kerr metric black holes. Nature 226, 64-65 (1970)

12. Bieri, L.: Black hole formation and stability: a mathematical investigation. Bull. Am. Math. Soc. (N.S.) 55, 1-30 (2018)

13. Burtscher, A.Y.: Initial data and black holes for matter models. In: Hyperbolic Problems: Theory, Numerics. Applications. AIMS Series in Applied Mathematics, vol 10, pp 336-345. American Institute of Mathematics, San Jose (2020)

14. Burtscher, A.Y., LeFloch, P.G.: The formation of trapped surfaces in spherically-symmetric Einstein-Euler spacetimes with bounded variation. J. Math. Pures Appl. 102, 1164-1217 (2014)

15. Butterfied, J.: Less is different: emergence and reduction reconciled. Found. Phys. 41, 1065-1135 (2011)

16. Candela, A.M., Flores, J.L., Sánchez, M.: Global hyperbolicity and Palais-Smale condition for action functionals in stationary spacetimes. Adv. Math. 218, 515-536 (2010)

17. Cardoso, V., Gualtieri, L.: Testing the black hole 'no-hair' hypothesis. Class. Quantum Gravity 33, 174001 (2016)

18. Carter, B.: Causal structure in space-time. Gen. Relativ. Gravit. 1, 349-391 (1971a)

19. Carter, B.: Axisymmetric black hole has only two degrees of freedom. Phys. Rev. Lett. 26, 331333 (1971b)

20. Carter, B. (1973). Black hole equilibrium states. Part I: Analytic and geometric properties of the Kerr solution. Black Holes-Les astres occlus, eds. De Witt, B., DeWitt-Morette, C., pp. 61-124 (Gordon and Breach). Reprinted in General Relativity and Gravitation 41, 2873-2938 (2009)

21. Carter, B.: The general theory of the mechanical, electromagnetic and thermodynamic properties of black holes. In: Hawking, S.W., Israel, W. (eds.) General Relativity: An Einstein Centenary Survey, pp. 294-369. Cambridge University Press, Cambridge (1979)

22. Carter, B.: Mathematical foundations of the theory of relativistic stellar and black hole configurations. In: Carter, B., Hartle, J.B. (eds.) Gravitation in Astrophysics (Cargèse 1986), pp. 63-122. Plenum Press, New York (1986)

23. Cederbaum, C.: Static Black Hole Uniqueness Theorems. Lectures 1-4. ICTP School of Geometry and Gravity (2019). https://www.youtube.com/watch?v=hf4qIiGVwLk

24. Chesler, P.M., Narayan, R., Curiel, E.: Singularities in Reissner-Nordström black holes. Class. Quantum Gravity 37, 025009 (2020)

25. Choquet-Bruhat, Y.: Hyperbolic partial differential equations on a manifold. Batelle Rencontres: 1967 Lectures in Mathematics and Physics, eds. C. DeWitt and J.A. Wheeler, pp. 84-106 (W.A. Benjamin) (1967) 
26. Choquet-Bruhat, Y.: General Relativity and the Einstein Equations. Oxford University Press, Oxford (2009)

27. Choquet-Bruhat, Y.: Beginnings of the Cauchy problem (2014). arXiv:1410.3490. Accessed on 23 Marc 2021

28. Choquet-Bruhat, Y.: A Lady Mathematician in this Strange Universe: Memoirs. World Scientific, Singapore (2018)

29. Choquet-Bruhat, Y., Geroch, R.: Global aspects of the Cauchy problem in general relativity. Commun. Math. Phys. 14, 329-335 (1969)

30. Christodoulou, D.: Reversible and irreversible transformations in black-hole physics. Phys. Rev. Lett. 25, 1596-1597 (1970)

31. Christodoulou, D.: The formation of black holes and singularities in spherically symmetric gravitational collapse. Commun. Pure Appl. Math. 44, 339-373 (1991)

32. Christodoulou, D.: On the global initial value problem and the issue of singularities. Class. Quantum Gravity 16, A23-A35 (1999a)

33. Christodoulou, D.: The instability of naked singularities in the gravitational collapse of a scalar field. Ann. Math. 149, 183-217 (1999b)

34. Christodoulou, D.: The Formation of Black Holes in General Relativity. European Mathematical Society, Zurich (2009)

35. Christodoulou, D., Klainerman, S.: The Global Nonlinear Stability of the Minkowski Space. Princeton University Press, Princeton (1993)

36. Chruściel, P.T.: On uniqueness in the large of solutions of Einstein's equations ("Strong Cosmic Censorship"). Mathematical Aspects of Classical Field Theory. Contemporary Mathematics 132, 235-274 (1992)

37. Chruściel, P.T.: Elements of causality theory (2011). arXiv:1110.6706. Accessed on 23 March 2021

38. Chruściel, P.T.: Elements of General Relativity. Springer, Cham (2019)

39. Chruściel, P.T.: Geometry of Black Holes. Oxford University Press, Oxford (2020)

40. Chruściel, P.T., Delay, E., Galloway, G.J., Howard, R.: Regularity of horizons and the area theorem. Ann. Henri Poincaré 2, 109-178 (2001)

41. Chruściel, P.T., Galloway, G.J., Pollack, D.: Mathematical general relativity: a sampler. Bull. Am. Math. Soc. 47, 567-638 (2010)

42. Chruściel, P.T., Isenberg, J.: Nonisometric vacuum extensions of vacuum maximal globally hyperbolic spacetimes. Phys. Rev. D 48, 1616-1628 (1993)

43. Chruściel, P.T., Isenberg, J., Moncrief, V.: Strong cosmic censorship in polarised Gowdy spacetimes. Class. Quantum Gravity 7, 1671-1680 (1990)

44. Chruściel, P.T., Lopes Costa, J., Heusler, M.: Stationary black holes: uniqueness and beyond. Living Rev. Relativ. 15(7), (2012). arXiv:1205.6112. Accessed on 23 March 2021

45. Chruściel, P.T., Paetz, T.T.: Characteristic initial data and smoothness of Scri. I. Framework and results. Ann. Henri Poincaré 16, 2131-2162 (2015)

46. Clarke, C.J.S.: The Analysis of Space-Time Singularities. Cambridge University Press, Cambridge (1993)

47. Coley, A.A.: Mathematical general relativity. Gen. Relativ. Gravit. 51, 78-112 (2019)

48. Curiel, E.: The analysis of singular spacetimes. Philos. Sci. 66, S119-S145 (1999). Revised and extended version at http://strangebeautiful.com/phil-phys.html

49. Curiel, E.: Singularities and Black Holes. The Stanford Encyclopedia of Philosophy (Spring 2019). https://plato.stanford.edu/archives/spr2019/entries/spacetime-singularities/

50. Curiel, E.: The many definitions of a black hole. Nat. Astron. 3, 27-34 (2019b)

51. Dafermos, M.: Stability and instability of the Cauchy horizon for the spherically symmetric Einstein-Maxwell-scalar field equations. Ann. Math. 158, 875-928 (2003)

52. Dafermos, M.: The formation of black holes in General Relativity [after D. Christodoulou] Séminaire Bourbaki 64, no. 1051 (2012). https://www.dpmms.cam.ac.uk/ md384/expose-chr.pdf

53. Dafermos, M.: The geometry and analysis of black hole spacetimes in general relativity (ETH Nachdiplom lectures) (2013). https://www.dpmms.cam.ac.uk/ md384/Ravello_Lectures_1.pdf

54. Dafermos, M.: Black holes without spacelike singularities. Commun. Math. Phys. 332, 729 (2014a)

55. Dafermos, M.: The mathematical analysis of black holes in general relativity. In: Proceedings of the ICM, 2014 (2014b). https://www.dpmms.cam.ac.uk/ md384/ICMarticleMihalis.pdf

56. Dafermos, M.: The cosmic censorship conjectures in classical general relativity (2017). https:// www.youtube.com/watch?v=ZBYAbejIvB4 
57. Dafermos, M.: The Cosmic Censorship Conjectures in General Relativity. ICTP School on Geometry and Gravity (2019). Lecture 1: https://www.youtube.com/watch?v=Lg1Cetf7V9I. Lecture 2: https://www.youtube.com/watch?v=SoRhBSt_mN0

58. Dafermos, M., Holzegel, G., Rodnianski, I.: The linear stability of the Schwarzschild solution to gravitational perturbations. Acta Math. 222, 1-214 (2019a)

59. Dafermos, M., Holzegel, G., Rodnianski, I.: Boundedness and decay for the Teukolsky equation on Kerr spacetimes I: the case $|a| \ll M$. Ann. PDE 5, 2 (2019b)

60. Dafermos, M., Luk, J.: The interior of dynamical vacuum black holes I: the $c^{0}$-stability of the Kerr Cauchy horizon (2017). arXiv:1710.01722. Accessed on 23 March 2021

61. Dafermos, M., Rodnianski, I.: Lectures on black holes and linear waves (2008). arXiv:0811.0354. Accessed on 23 March 2021

62. Dias, O.J.C., Reall, H.S., Santos, J.E.: Strong cosmic censorship: taking the rough with the smooth. J. High Energy Phys. (2018). https://doi.org/10.1007/JHEP10(2018)001

63. Doboszewski, J.: Non-uniquely extendible maximal globally hyperbolic spacetimes in classical general relativity: a philosophical survey. Eur. Stud. Philos. Sci. 6, 193-212 (2017)

64. Doboszewski, J.: Relativistic spacetimes and definitions of determinism. Eur. J. Philos. Sci. 9, 24 (2019). https://doi.org/10.1007/s13194-019-0248-6

65. Doboszewski, J.: Epistemic holes and determinism in classical general relativity. Br. J. Philos. Sci. 71, 1093-1111 (2020)

66. Earman, J.: Bangs, Crunches, Whimpers, and Shrieks: Singularities and Acausalities in Relativistic Spacetimes. Oxford University Press, Oxford (1995)

67. Earman, J.: Tolerance for spacetime singularities. Found. Phys. 26, 623-640 (1996)

68. Earman, J.: The Penrose-Hawking singularity theorems: history and implications. In: Goenner, H., Renn, J., Ritter, T., Sauer, T. (eds.) The Expanding Worlds of General Relativity (Einstein Studies), vol. 7, pp. 236-267. Birkhäuser, Boston (1999)

69. Earman, J.: Curie's Principle and spontaneous symmetry breaking. Int. Stud. Philos. Sci. 18, 173198 (2004)

70. Earman, J.: Aspects of determinism in modern physics. In: Butterfield, J., Earman, J. (eds.) Handbook of the Philosophy of Science: Philosophy of Physics, Part B, vol. 2, pp. 1369-1434. NorthHolland/Elsevier, New York (2007)

71. Earman, J., Eisenstaedt, J.: Einstein and singularities. Stud. Hist. Philos. Mod. Phys. 30, 185-235 (1999)

72. Einstein, A.: Die Feldgleichungen der Gravitation, pp. 844-847. Sitzungsberichte der Königlich Preußischen Akademie der Wissenschaften, Berlin (1915)

73. Einstein, A.: Über Gravitationswellen, pp. 154-167. Sitzungsberichte der Königlich Preußschen Akademie der Wissenschaften, Berlin (1918)

74. Einstein, A.: In: Janssen, M., et al. (eds)The Collected Papers of Albert Einstein: The Berlin Years: Writings, 1918-1921, vol. 7. Princeton University Press (2002). https://einsteinpapers.press.princ eton.edu/vol7-doc/

75. Eisenstaedt, J.: Lemaître and the Schwarzschild solution. In: Earman, J., Janssen, M., Norton, J.D. (eds.) The Attraction of Gravitation: New Studies in the History of General Relativity, pp. 353389. Birkhäuser, Boston (1993)

76. Ellis, G.F.R.: Stephen Hawking's 1966 Adams Prize Essay. Eur. J. Phys. H 39, 403-411 (2014)

77. Event Horizon Telescope Collaboration: First M87 event horizon telescope results. V. Physical origin of the asymmetric ring. Astrophys. J. Lett. 875(L5,) 1-31 (2019)

78. Falcke, H.: Licht im Dunkeln. Klett-Cotta, Stuttgart (2020)

79. Finkelstein, D.: Past-future asymmetry of the gravitational field of a point particle. Phys. Rev. 110, 956-967 (1958)

80. Franklin, A.D.: Is seeing believing? Observation in physics. Phys. Perspect. 19, 321-423 (2017)

81. Frauendiener, J.: Conformal infinity. Living Rev. Relativ. 3, 4 (2000)

82. Friedrich, H.: Smoothness at null infinity and the structure of initial data. In: Friedrich, H., Chruściel, P.T. (eds.) The Einstein Equations and the Large Scale Behavior of Gravitational Fields, pp. 121-203. Springer, Basel (2004)

83. Friedrich, H.: Editorial note to: Roger Penrose, conformal treatment of infinity. Gen. Relativ. Gravit. 43, 897-900 (2011)

84. Friedrich, H.: Peeling or not peeling-is that the question? Class. Quantum Gravity 35, 083001 (2018) 
85. Gajic, D., Luk, J.: The interior of dynamical extremal black holes in spherical symmetry. Pure Appl. Anal. 1, 263-326 (2019)

86. Geroch, R.: What is a singularity in General Relativity? Ann. Phys. (N.Y.) 48, 526-540 (1968)

87. Geroch, R.: Domain of dependence. J. Math. Phys. 11, 437-449 (1970)

88. Geroch, R.: Asymptotic structure of space-time. In: Esposito, F.P., Witten, L. (eds.) Asymptotic Structure of Space-Time, pp. 1-105. Plenum, New York (1977)

89. Geroch, R., Horowitz, G.: Asymptotically simple does not imply asymptotically Minkowskian. Phys. Rev. Lett. 40, 203-206 (1978)

90. Geroch, R., Kronheimer, E.H., Penrose, R.: Ideal points in space-time. Proc. R. Soc. (Lond.) A327, 545-567 (1972)

91. Giorgi, E., Klainerman, S., Szeftel, J.: A general formalism for the stability of Kerr (2020). arXiv: 2002.02740. Accessed on 23 March 2021

92. Godart, O.: Contributions of Lemaitre to general relativity (1922-1934). In: Eisenstaedt, J., Kox, A.J. (eds.) Studies in the History of General Relativity, pp. 437-452. Birkhäuser, Boston (1992)

93. Goenner, H.F.M.: On the history of unified field theories. Living Rev. Relativ. 7, 2 (2004)

94. Gundlach, C., Martin-Garcia, J.M.: Critical phenomena in gravitational collapse. Living Rev. Relativ. 10, 5 (2007)

95. Hawking, S.W.: Singularities and the geometry of spacetime (Adams Prize Essay) (1966). Reprinted in Eur. J. Phys. H 39, 413-503 (2014)

96. Hawking, S.W.: Gravitational radiation from colliding black holes. Phys. Rev. Lett. 26, 1344-1346 (1971)

97. Hawking, S.W.: Black holes in general relativity. Commun. Math. Phys. 25, 152-166 (1972)

98. Hawking, S.W., Ellis, G.F.R.: The Large Scale Structure of Space-Time. Cambridge University Press, Cambridge (1973)

99. Heusler, M.: Black Hole Uniqueness Theorems. Cambridge University Press, Cambridge (1996)

100. Hilbert, D.: Die Grundlagen der Physik (Zweite Mitteilung). Nachrichten von der Königlichen Gesellschaft der Wissenschaften zu Göttingen, Mathematisch-Physikalische Klasse, pp 53-76 (1917)

101. Hintz, P., Vasy, A.: The global non-linear stability of the Kerr-de Sitter family of black holes. Acta Math. 220, 1-206 (2018)

102. Hiscock, W.A.: Evolution of the interior of a charged black hole. Phys. Lett. A 83, 110-112 (1981)

103. Hodges, A.: Extra Time: Professor Sir Roger Penrose in conversation with Andrew Hodges (Part 2) (2014). https://www.youtube.com/watch?v=zN5eLsI_Tuo (part 1); https://www.youtube.com/ watch? $\mathrm{v}=\mathrm{FFWbpHml11 \textrm {g }}$

104. Hounnonkpe, R.A., Minguzzi, E.: Globally hyperbolic spacetimes can be defined without the 'causal' condition (2019). arXiv:1908.11701. Accessed on 23 March 2021

105. Ionescu, A., Klainerman, S.: Rigidity results in general relativity: a review (2015). arXiv:1501. 01587. Accessed on 23 March 2021

106. Israel, W.: Event horizons in static vacuum space-times. Phys. Rev. 164, 1776-1779 (1967)

107. Israel, W.: Event horizons in static electrovac space-times. Commun. Math. Phys. 8, 245-260 (1968)

108. Israel, W.: Event horizons and gravitational collapse. Gen. Relativ. Gravit. 2, 53-59 (1971)

109. Israel, W.: Dark stars: the evolution of an idea. In: Hawking, S.W., Israel, W. (eds.) Three Hundred Years of Gravitation, pp. 199-276. Cambridge University Press, Cambridge (1987)

110. Joshi, P.S.: Global Aspects in Gravitation and Cosmology. Oxford University Press, Oxford (1993)

111. Joshi, P.S.: Gravitational Collapse and Spacetime Singularities. Cambridge University Press, Cambridge (2007)

112. Kerr, R.: Gravitational field of a spinning mass as an example of algebraically special metrics. Phys. Rev. Lett. 11, 237-238 (1963)

113. Klainerman, S.: Are black holes real? (2014) https://www.youtube.com/watch?v=zj1QkhvHVGU

114. Klainerman, S., Luk, J., Rodnianski, I.: A fully anisotropic mechanism for formation of trapped surfaces. Invent. Math. 198, 1-26 (2014)

115. Klainerman, S., Nicolò, F.: Peeling properties of asymptotically flat solutions to the Einstein vacuum equations. Class. Quantum Gravity 20, 3215-3258 (2003)

116. Klainerman, S., Rodnianski, I.: On the formation of trapped surfaces. Acta Math. 208, 211-333 (2012)

117. Klainerman, S., Rodnianski, I., Szeftel, J.: The bounded $L^{2}$ curvature conjecture. Invent. Math. 202, 91-216 (2015) 
118. Klainerman, S., Szeftel, J.: Global nonlinear stability of Schwarzschild spacetime under polarized perturbations (2017). arXiv:1711.07597. Accessed on 23 March 2021

119. Królak, A.: Towards the proof of the cosmic censorship hypothesis. Class. Quantum Gravity 3, 267-280 (1986)

120. Królak, A.: Nature of singularities in gravitational collapse. Prog. Theor. Phys. Suppl. 136, 45-56 (1999)

121. Królak, A.: Cosmic censorship hypothesis. Contemp. Math. 359, 51-64 (2004)

122. Landsman, K.: Foundations of Quantum Theory: From Classical Concepts to Operator Algebras. Springer, Cham (2017). https://www.springer.com/gp/book/9783319517766

123. Leray, J.: Hyperbolic Differential Equations. Mimeographed Lecture Notes. The Institute for Advanced Study (1953)

124. Li, J., Yu, P.: Construction of Cauchy data of vacuum Einstein field equations evolving to black holes. Ann. Math. 181, 699-768 (2015)

125. Lightman, A.: AIP Oral History Interviews: Roger Penrose (1989). https://www.aip.org/historyprograms/niels-bohr-library/oral-histories/34322

126. Luk, J.: Weak null singularities in general relativity. J. AMS 31, 1-63 (2017)

127. Luk, J., Oh, S.-J.: Strong cosmic censorship in spherical symmetry for two-ended asymptotically flat Initial data I: the interior of the black hole region. Ann. Math. 190, 1-111 (2019a)

128. Luk, J., Oh, S.-J.: Strong cosmic censorship in spherical symmetry for two-ended asymptotically flat Initial data II: the exterior of the black hole region. Ann. PDE 5(6) (2019b)

129. Luk, J., Sbierski, J.: Instability results for the wave equation in the interior of Kerr black holes. J. Funct. Anal. 271, 1948-1995 (2016)

130. Luminet, J.P.: Black Holes. Cambridge University Press, Cambridge (1992)

131. Manchak, J.B.: What is a physically reasonable spacetime? Philos. Sci. 78, 410-420 (2011)

132. Manchak, J.B.: On the inextendibility of space-time. Philos. Sci. 84, 1215-1225 (2017)

133. Melia, F.: Cracking the Einstein Code: Relativity and the Birth of Black Hole Physics. University of Chicago Press, Chicago (2009)

134. Minguzzi, E.: Limit curve theorems in Lorentzian geometry (2008). arXiv:0712.3942. Accessed on 23 March 2021

135. Minguzzi, E.: Lorentzian causality theory. Living Rev. Relativ. 22, 3 (2019)

136. Misner, C.W., Thorne, K.S., Wheeler, J.A.: Gravitation. Freeman, Reading (1973)

137. Moncrief, V.: Global properties of Gowdy spacetimes with $T^{3} \times \mathbb{R}$ topology. Ann. Phys. (N.Y.) 132, 87-107 (1981)

138. Moncrief, V., Eardley, D.M.: The global existence problem and cosmic censorship in general relativity. Gen. Relativ. Gravit. 13, 887-892 (1981)

139. O’Neill, B.: Semi-Riemannian Geometry. Academic, New York (1983)

140. Ong, Y.C.: Space-time singularities and cosmic censorship conjecture: a review with some thoughts. Int. J. Mod. Phys. A 35, 2030007 (2020)

141. Paetz, T.T.: Characteristic initial data and smoothness of Scri. II. Asymptotic expansions and construction of conformally smooth data sets. J. Math. Phys. 55, 102503 (2014)

142. Penrose, R.: Null hypersurface initial data for classical fields of arbitrary spin and for general relativity. Aerospace Research Laboratories, pp 63-65 (1963). Reprinted in Gen. Relativ. Gravit. 12, 225-264 (1980)

143. Penrose, R.: Conformal treatment of infinity. Relativity, Groups, and Topology, eds. B. DeWitt and C.M. DeWitt-Morette, pp. 565-584 (Gordon \& Breach) (1964). Reprinted in Gen. Relativ. Gravit. 43, 901-922 (2011), with a historical introduction by H. Friedrich, ibid., pp. 897-900

144. Penrose, R.: Gravitational collapse and space-time singularities. Phys. Rev. Lett. 14, 57-59 (1965)

145. Penrose, R. (1966). An analysis of the structure of space-time. Adams Prize Essay. In: Roger Penrose: Collected Works: 1953-1967, vol 1, pp. 579-730. Oxford University Press, Oxford (2011)

146. Penrose, R.: Structure of space-time. In: DeWitt, C., Wheeler, J.A. (eds.) Batelle Rencontres: 1967 Lectures in Mathematics and Physics, pp. 121-235. W.A. Benjamin, San Francisco (1968)

147. Penrose, R.: Gravitational collapse: the role of general relativity. Rivista del Nuovo Cimento, Numero Speziale I, 252 (1969). Reprinted in Gen. Relativ. Gravit. 34, 1141-1165 (2002)

148. Penrose, R.: Techniques of Differential Topology in Relativity. SIAM, Philadelphia (1972)

149. Penrose, R.: Singularities and time-asymmetry. In: Hawking, S.W., Israel, W. (eds.) General Relativity: An Einstein Centenary Survey, pp. 581-638. Cambridge University Press, Cambridge (1979)

150. Penrose, R.: The question of cosmic censorship. J. Astrophys. Astron. 20, 233-248 (1999) 
151. Penrose, R., Floyd, R.: Extraction of rotational energy from a black hole. Nature 229, 177-179 (1971)

152. Penrose, R., Rindler, W.: Spinors and Space-Time Spinor and Twistor Methods in Space-Time: Geometry, vol. 2. Cambridge University Press, Cambridge (1986)

153. Poisson, E.: A Relativist's Toolkit: The Mathematics of Black-Hole Mechanics. Cambridge University Press, Cambridge (2004)

154. Reichenbach, H.: Axiomatik der relativistischen Raum-Zeit-Lehre. Vieweg, Wiesbaden (1924)

155. Rindler, W.: Visual horizons in world models. Mon. Not. R. Astron. Soc. 116, 662-677 (1956)

156. Ringström, H.: The Cauchy Problem in General Relativity. European Mathematical Society, Zurich (2009)

157. Ringström, H.: Origins and development of the Cauchy problem in general relativity. Class. Quantum Gravity 32, 124003 (2015)

158. Robb, A.A.: A Theory of Time and Space. Cambridge University Press, Cambridge (1914)

159. Robb, A.A.: Geometry of Time and Space. Cambridge University Press, Cambridge (1936)

160. Robinson, D.C.: Four decades of black hole uniqueness theorems. In: Wiltshire, D., Visser, M., Scott, S.M. (eds.) The Kerr Spacetime: Rotating Black Holes in General Relativity, pp. 114-115. Cambridge University Press, Cambridge (2009)

161. Ruffini, R., Wheeler, J.A.: Relativistic cosmology and space platforms. In: Moore, A.F., Hardy, V. (eds) The Significance of Space Research for Fundamental Physics, pp. 45-171. European Space Research Organization (1971a). http://inis.iaea.org/collection/NCLCollectionStore/_Public/03/024/ 3024932.pdf

162. Ruffini, R., Wheeler, J.A.: Introducing the black hole. Phys. Today 24(January), 30-41 (1971b)

163. Russell, B.: Introduction to Mathematical Philosophy, 2nd edn. Allen \& Unwin, Sydney (1920)

164. Saari, D.G., Xia, Z.: Off to infinity in finite time. Not. AMS 42, 538-546 (1995)

165. Sanders, R.H.: Revealing the Heart of the Galaxy: The Milky Way and Its Black Hole. Cambridge University Press, Cambridge (2014)

166. Sbierski, J.: On the existence of a maximal Cauchy development for the Einstein equations: a dezornification. Ann. Henri Poincaré 17, 301-329 (2016)

167. Sbierski, J.: The $C^{0}$-inextendibility of the Schwarzschild spacetime and the spacelike diameter in Lorentzian geometry. J. Differ. Geom. 108, 319-378 (2018a)

168. Sbierski, J.: On the proof of the $C^{0}$-inextendibility of the Schwarzschild spacetime. J. Phys. Conf. Ser. 968, 012012 (2018b)

169. Schoen, R., Yau, S.T.: The existence of a black hole due to condensation of matter. Commun. Math. Phys. 65, 575-579 (1983)

170. Senovilla, J.M.M.: Singularity theorems and their consequences. Gen. Relativ. Gravit. 29, 701-848 (1997). Corrected version: arXiv:1801.04912. Accessed on 23 March 2021

171. Senovilla, J.M.M., Garfinkle, D.: The 1965 Penrose singularity theorem. Class. Quantum Gravity 32, 124008 (2015)

172. Simpson, M., Penrose, R.: Internal instability in a Reissner-Nordström black hole. Int. J. Theor. Phys. 7, 183-197 (1973)

173. Stachel, J.: The Cauchy problem in general relativity - the early years. In: Eisenstaedt, J., Kox, A.J. (eds.) Studies in the History of General Relativity, pp. 407-418. Birkhäuser, Boston (1992)

174. Stewart, J.: Advanced General Relativity. Cambridge University Press, Cambridge (1991)

175. Thorne, K.S.: Black Holes and Time Warps: Einstein's Outrageous Legacy. W.W. Norton, New York (1994)

176. Tipler, F.J., Clarke, C.J.S., Ellis, G.F.R.: Singularities and horizons-a review article. In: Held, A. (ed.) General Relativity and Gravitation: One Hundred Years After the Birth of Albert Einstein, vol. 2, pp. 97-206. Plenum Press, New York (1980)

177. Valiente Kroon, J.: Conformal Methods in General Relativity. Cambridge University Press, Cambridge (2016)

178. Van de Moortel, M.: The breakdown of weak null singularities inside black holes (2020). arXiv: 1912.10890. Accessed on 23 March 2021

179. Wald, R.M.: General Relativity. University of Chicago Press, Chicago (1984)

180. Weinberg, S.: Lectures on Astrophysics. Cambridge University Press, Cambridge (2020)

181. Weyl, H.: Die Idee der Riemannschen Fläche. Teubner, Leipzig (1913)

182. Weyl, H.: Raum - Zeit - Materie: Vorlesungen über Allgemeine Relativitätstheorie (Springer) (1918a). English translation (of the fourth edition from 1921): Space Time Matter (Methuen, 1922)

183. Weyl, H.: Reine Infinitesimalgeometrie. Math. Z. 2, 384-411 (1918b) 
184. Wright, A.S.: The origins of Penrose diagrams in physics, art, and the psychology of perception. Endeavour 37, 133-139 (2013)

185. Wright, A.S.: The advantages of bringing infinity to a finite place: Penrose diagrams as objects of intuition. Hist. Stud. Nat. Sci. 44, 99-139 (2014)

186. Xia, Z.: The existence of non-collision singularities in Newtonian systems. Ann. Math. 135, 411468 (1992)

187. Zeeman, E.C.: Causality implies the Lorentz group. J. Math. Phys. 5, 490-493 (1964)

Publisher's Note Springer Nature remains neutral with regard to jurisdictional claims in published maps and institutional affiliations. 